Nonl. Analysis and Differential Equations, Vol. 2, 2014, no. 1, 1 - 44

HIKARI Ltd, www.m-hikari.com

http://dx.doi.org/10.12988/nade.2014.3615

\title{
Multiplicity Results and Global Bifurcations for a Degenerate Elliptic Equation
}

\author{
M. Amattat
}

CFIE-Rabat, Morocco

Copyright (C) 2013 M. Amattat. This is an open access article distributed under the Creative Commons Attribution License, which permits unrestricted use, distribution, and reproduction in any medium, provided the original work is properly cited.

\begin{abstract}
We consider the question of determining the exact number of solutions of the problem $E_{\lambda}^{p}$ below We distinguish two cases: whether $1<p \ll 2$ or $2<p<\infty$. In the first case, we shall show that the problem $E_{\lambda}^{p}$ behaves like in the semi-linear case which corresponds to $p=2$.. In the second case and under some conditions which will be specified later; we shall show that the spectrum for problem $E_{\lambda}^{p}$ consists of a collection of intervals $I_{n}, n=1,2,3$, whose ends points are members of the sequences $\left(\lambda_{n}\right)_{n},\left(\mu_{n}\right)_{n}$ where the first one is the sequence of eigenvalues for the pseudo-Laplacian operator and the other one is a sequence of a kind of eigenvalues for $E_{\lambda}^{p}$. And each time $\lambda=\mu_{n}$, there exists secondary bifurcating continuum $\varepsilon_{n}$ of singular solutions which are diffeomorphic to $[0, \pi]^{n}, n=1,2$. .
\end{abstract}

Keywords: Degenerate Elliptic Equation - Pseudo-Laplacian operator Global Bifurcations

\section{Introduction}

Let us consider the Dirichlet's problem associated with nonlinear degenerate elliptic equation. $E_{\lambda}^{p}$

$$
\begin{array}{lr}
-\frac{d}{d x}\left(\left|\frac{d u}{d x}\right|^{p-2} \frac{d u}{d x}\right)=\lambda f(u) ; & x \in] 0, \pi[ \\
u(0)=u(\pi)=0,1<p<+\infty, & \lambda \in \mathbb{R}
\end{array}
$$


We investigate the non trivial solutions of this problem and more particularly we consider the question of determining the exact number of solutions of this problem. We distinguish two cases: whether $0 \ll p \ll 2$ or $2<p<\infty$. In the first case, we shall show that the problem $E_{\lambda}^{p}$ behaves like in the semi-linear case which corresponds to $p=2$. Indeed, we shall show that bifurcation can appear in the point $(0, \lambda)$ only it $\lambda$ belongs to the set $\left\{\lambda_{k}\right\}_{k=1}^{+\infty}$, where

$$
\lambda_{n}=\frac{2^{p} n^{p}(p-1)}{\pi^{p} p^{p}} B^{p}\left(\frac{1}{p}, 1-\frac{1}{p}\right)
$$

is the eigenvalue of the problem $\wp(\lambda)$

$$
\begin{array}{ll}
-\frac{d}{d x}\left(\left|\frac{d u}{d x}\right|^{p-2} \frac{d u}{d x}\right)=\lambda|u|^{p-2} u ; & x \in] 0, \pi[ \\
u(0)=u(\pi)=0, \quad 1<p<+\infty, & \lambda \in \mathbb{R}^{+}
\end{array}
$$

For more details about the problem $\wp(\lambda)$ the reader is referred to see [1].

We shall always refer to the first case as the semi-linear case and to the second case as the superlinear case. It is interesting to see what becomes of the notion of point spectrum of $E_{\lambda}^{p}$ when one goes from the semi-linear to the superlinear case. In the superlinear case and under some conditions which will be precise later : we shall show that the spectrum for problem $E_{\lambda}^{p}$ consists of a collection of intervals $I_{n}, n=1,2,3 \ldots$, whose ends points are members of the sequences $\left\{\lambda_{n}\right\},\left\{\mu_{n}\right\}$ where the first one is the sequence of eigenvalues for $\wp(\lambda)$ and the other one is a sequence of a kind of eigenvalues for $E_{\lambda}^{p}$. And each time $\lambda=\mu_{n}$, there exists secondary bifurcating continuum $\varepsilon_{n}$ of singular solutions which are diffeomorphic to $[0, \pi]^{n}, n=1,2 \ldots$

The approach used in this paper is based only on the elementary tools of the theory or ordinary differential equations and essentially the use of a similar type of the time map for problem $E_{\lambda}^{p}$.

Even there has been a significant amount of work that has been done on degenerate elliptic equations, and in particular, bifurcation problems for $\mathrm{p}$ Laplacian equations, not only for one-dimensional problems but in higher dimensions as well, in the last 25 years. Authors who have contributed to this field include, for instance, Laurent Veron, M.Guedda [16], Pavel Drabek [3], [8], [9], [10], [11], [12], Bryan Rynne [4], [5], [13], [14], [15], and many others. However, until recently, the basic spectral properties obtained in the case $p>2$ and the exact numbers of solutions of problem $E_{\lambda}^{p}$ and especially the existence of collection of intervals $I_{n}, n=1,2,3 \ldots$ had not been obtained.

Our study has been motivated by the work done by Chaffee and Infantee in the semi-linear case [6]. 
They considered the following problem: $\left(E_{\lambda}^{2}\right)$

$$
\begin{aligned}
& \left.u^{\prime \prime}=\lambda f(u) \quad x \in\right] 0, \pi[ \\
& u(0)=u(\pi)=0 \quad \lambda>0
\end{aligned}
$$

Where $f$ is a nonlinearity which satisfies the following hypotheses:

$$
\begin{aligned}
\left.\bar{H}_{1}\right) & : f: \mathbb{R} \rightarrow \mathbb{R} \text { is of classe } C^{2} \\
\left.\bar{H}_{2}\right) & : f(0)=0 \\
\left.\bar{H}_{3}\right) & : f^{\prime}(0)>0 \\
\left.\bar{H}_{4}\right) & : \lim _{|u| \rightarrow \infty} \sup f(u) u^{-1}<0 \\
\left.\bar{H}_{5}\right) & : \operatorname{sgn} f^{\prime \prime}=-\operatorname{sgn} u
\end{aligned}
$$

They obtain the following results

\section{Theorem}

i If $\lambda \leq \frac{1}{f^{\prime}(0)}$, then problem $\left(E_{\lambda}^{2}\right)$ has only the trivial solution;

ii If $\frac{n^{2}}{f^{\prime}(0)}<\lambda \leq \frac{(n+1)^{2}}{f^{\prime}(0)}, n=1,2, \ldots$, then problem $\left(E_{\lambda}^{2}\right)$ has precisely $2 n+1$ solutions.

Before passing to the study of the problem $\left(E_{\lambda}^{2}\right)$ in the next section, we now state explicitly the main's theorems.

As has been said above, we shall show the striking difference between the nonlinear $(p \neq 2)$ and semi-linear $p=2$ case.

We consider a nonlinearity $f$ which satisfies the following hypotheses:

\section{Hypotheses governing the function $f$.}

Throughout the remaining part of this work we shall assume that the function $f$ appearing in $\left(E_{\lambda}^{p}\right)$ satisfies the following hypotheses:

$$
\begin{aligned}
\bar{H}_{1} & : f: \mathbb{R} \rightarrow \mathbb{R} \text { is at least of classe } C^{1} \text { for } u \neq 0 \\
\left.\bar{H}_{2}\right) & : f(0)=0 \\
\left.\bar{H}_{3}\right) & : \lim _{u \rightarrow 0} \frac{f(u)}{|u|^{p-2} u}=\alpha>0 \\
\left.\bar{H}_{4}\right) & : \lim _{|u| \rightarrow \infty} \sup \frac{f(u)}{|u|^{p-2} u} \leq 0 \\
\left.\bar{H}_{5}\right) & :\left(\frac{F(u)}{f(u)}\right)^{\prime}>\frac{1}{p} ; \forall u \in\left(a_{-}, a_{+}\right) \text {avec } u \neq 0
\end{aligned}
$$


Where $\left(a_{-}, a_{+}\right)$is the maximal interval centered on the origin such that:

$$
f(u) \neq 0 ;-\infty \leq a_{-}<0<a_{+} \leq+\infty
$$

and

$$
F(u)=\int_{0}^{u} f(\xi) d \xi ; u \in \mathbb{R}
$$

Furthermore, we suppose in case $a_{-}$and $a_{+}$are finite that

$$
\left.\bar{H}_{6}\right) \quad f(u) u<0 \quad \forall u \notin\left(a_{-}, a_{+}\right)
$$

\section{Remark}

1. In case both $a_{-}$and $a_{+}$are finite, the condition $\left.\bar{H}_{6}\right)$ implies $\left.\bar{H}_{4}\right)$;

2. When $p=2$, one can replace the condition $\bar{H}_{5}$ ) by a sufficient condition, namely:

$$
\left.\overline{\bar{H}}_{5}\right) \quad \operatorname{sgn} f^{\prime \prime}(u)=-\operatorname{sgn} u \quad \forall u \in\left(a_{-}, a_{+}\right)
$$

\section{Examples of function $\mathbf{f}$}

Here is some examples of functions which satisfy the above conditions:

1. $f(u)=-|u|^{p} u+|u|^{p-1} u ; p>1$

2. $f(u)=-|u|^{2(p-1)} u+|u|^{p-1} u ; p>1$

\section{Summary of the main results}

We shall distinguish two cases: $p \leq 2$ and $p>2$.

\section{First case A: $1<p \leq 2$}

\section{Theorem 1}

i If $\lambda \leq \frac{\lambda_{1}}{\alpha}$,

then problem $\left(E_{\lambda}^{p}\right)$ has only the trivial solution

ii If $\frac{\lambda_{n}}{\alpha}<\lambda \leq \frac{\lambda_{n+1}}{\alpha}, n=1,2, \ldots$

then problem $\left(E_{\lambda}^{p}\right)$ has precisely $2 n+1$ solutions where $\lambda_{n}$ is the eigenvalue of $(\wp(\lambda))$ and $\alpha$ is given by $\left.\bar{H}_{3}\right)$

Thus under the first case A; the bifurcation picture is as portrayed in figure 1. 




Figure 1: Where $u(\xi)=\max _{x \in] 0, \pi[}|u(x)|$

\section{Second case B: $p>2$}

\section{Definition 1}

By a regular solution of problem $E_{\lambda}^{p}$, we mean every solution $u$ which has a finite number of zeros in $(0, \pi)$, and whose first derivative vanishes only once between two consecutive zeros.

\section{Definition 2}

By a singular solution of problem $E_{\lambda}^{p}$, we mean every solution $u$ which has a finite number of zero in $(0, \pi)$, and such that between two consecutive zeros, the first derivative vanishes at least in a subinterval.

\section{Remark}

We will show later that there are only those two kinds of solutions. Next, we will treat three sub cases which go with the following additional hypotheses:

i $a_{ \pm}$are both infinite;

ii $a_{ \pm}$are both finite, and the two following integrals are infinite

$$
\begin{gathered}
\int_{0}^{a_{+}}\left\{F\left(a_{+}\right)-F(u)\right\}^{-\frac{1}{p}} d u=+\infty \\
\int_{a_{-}}^{0}\left\{F\left(a_{-}\right)-F(u)\right\}^{-\frac{1}{p}} d u=+\infty
\end{gathered}
$$

iii $f$ is odd, $\left|a_{-}\right|=\left|a_{+}\right|=a$, is finite and the two following integrals are finite

$$
\int_{0}^{a_{+}}\{F(a)-F(u)\}^{-\frac{1}{p}} d u<+\infty
$$

Under the second case $B$ and the statements $i$ ) or $i i$ ) we obtain: 


\section{Theorem 2}

i) If $\lambda \leq \frac{\lambda_{1}}{\alpha}$ then problem $E_{\lambda}^{p}$

has only the trivial solution

ii) If $\frac{\lambda_{1}}{\alpha}<\lambda \leq \frac{\lambda_{n+1}}{\alpha}, n=1,2, \ldots$

then problem $E_{\lambda}^{p}$ has precisely $2 n+1$ regular solutions and no singular solutions:

$$
\begin{aligned}
& u_{0}(x) \equiv 0, \quad u_{n}^{ \pm}(x) \text { where } \\
& \frac{d u_{n}^{+}}{d x}(0)>0, \quad \frac{d u_{n}^{-}}{d x}(0)<0
\end{aligned}
$$

and $u_{n}^{ \pm}(x)$ has exactly $n-1$ simple zeros in $(0, \pi)$.

Thus under the second case $B$ and the statements $i$ ) or $i i$ ) above, the bifurcation picture is as portrayed in figure 2 .

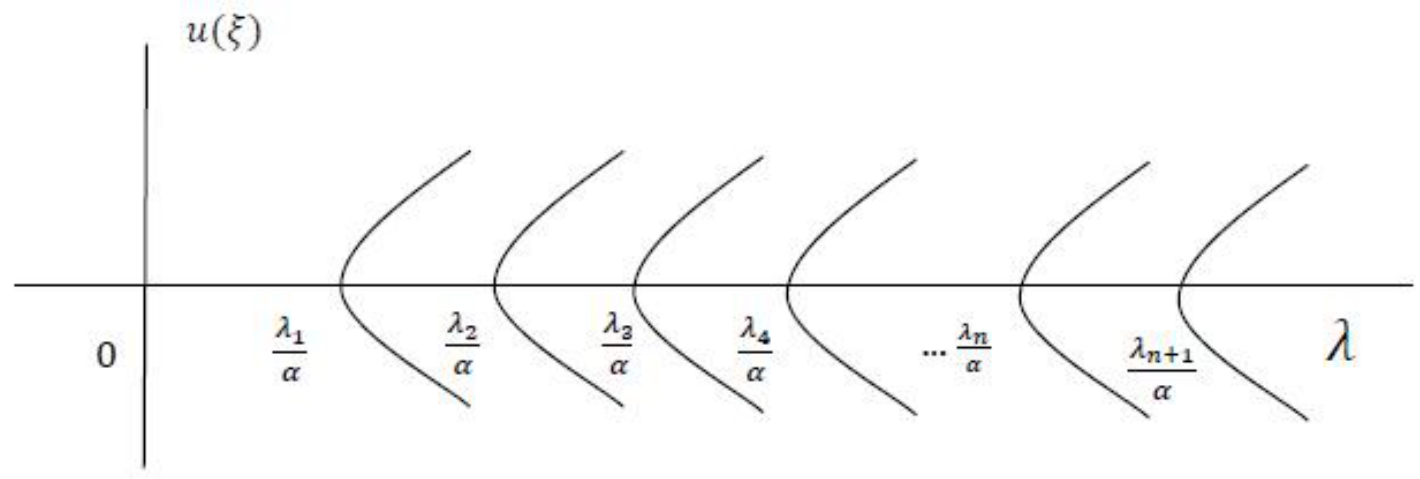

Figure 2: $u(\xi)=\max _{x \in] 0, \pi[}|u(x)|$

We notice that under the first case $A$ and under the second case $B$ and the statements $i$ ) or $i i$ ), the problem $E_{\lambda}^{p}$ behaves like in the semi-linear case $(p \leq 2)$.

Under the statement $i i i$ ), we consider the following real number whose significance only appears later:

$$
r(p, F)=\frac{1}{p^{\frac{p-1}{p}} \alpha^{\frac{1}{p}} \frac{T(F)}{B\left(\frac{1}{p}, 1-\frac{1}{p}\right)}-1}
$$


where

$$
T(F)=\int_{0}^{a}\{F(a)-F(u)\}^{-\frac{1}{p}} d u ; \quad a=-a_{-}=a_{+}
$$

Under The second case $B$, and $i i i)$, we shall distinguish two cases depending on whether:

$$
r(p, F)<1 \text { or } r(p, F) \geq 1
$$

First case: $r(p, F)<1$

We obtain

\section{Theorem 3}

i) If $\lambda \leq \frac{\lambda_{1}}{\alpha}$,

Then problem $E_{\lambda}^{p}$ has only the trivial solution

ii) If $\frac{\lambda_{1}}{\alpha}<\lambda \leq \frac{\lambda_{2}}{\alpha}$

Then problem $E_{\lambda}^{p}$ has precisely three regular solutions and no singular solutions

iii) If $\frac{\lambda_{n+1}}{\alpha}<\lambda<\mu_{n} \quad n=1,2, \ldots$

Then problem $E_{\lambda}^{p}$ has precisely five regulars solutions

iv) If $\mu_{n}<\lambda \leq \frac{\lambda_{n+2}}{\alpha} \quad n=1,2, \ldots$

Then problem $E_{\lambda}^{p}$ has precisely three regular solutions and each time $\lambda=\mu_{n}$ there exists a secondary bifurcating continuum $\varepsilon_{n}$ of non trivial singular solutions which are diffeomorphic to $] 0, \pi^{n}, n=1,2, \ldots$ where

$$
\mu_{n}=\frac{2^{p} n^{p}(p-1)}{\pi^{p} p}\left(\int_{0}^{a}\{F(a)-F(u)\}^{-\frac{1}{p}} d u\right)^{p}
$$

Second case: $r(p, F) \geq 1$

In this case, we have:

\section{Theorem 4}

i) $\lambda \leq \frac{\lambda_{1}}{\alpha}$,

Then problem $E_{\lambda}^{p}$ has only the trivial solution;

ii) If $\frac{\lambda_{n}}{\alpha}<\lambda<\mu_{n} \quad n=1,2, \ldots,[r(p, F)]$

Then problem $E_{\lambda}^{p}$ has precisely three regular solutions; 
iii) If $\mu_{n}<\lambda \leq \frac{\lambda_{n+1}}{\alpha} \quad n=1,2, \ldots,[r(p, F)]$

Then problem $E_{\lambda}^{p}$ has no regular solutions (has only the trivial solution), on the other hand there is an apparition of continuum $\varepsilon_{n}$ of singular solutions which are diffeomorphic to $[0, \pi]^{n}, n=1,2, \ldots$

If $\frac{\lambda_{n+1}}{\alpha}<\lambda<\frac{\lambda_{n+2}}{\alpha}$

Then problem $E_{\lambda}^{p}$ has precisely three regular solutions.

iv If $\frac{\lambda_{n+1}}{\alpha} \leq \lambda<\mu_{n} \quad n>n^{*}=[r(p, F)]$

Then problem $E_{\lambda}^{p}$ has precisely five regular solutions

$\mathbf{v}$ And if $\mu_{n}<\lambda \leq \frac{\lambda_{n+1}}{\alpha} \quad n=n^{*}+1, \ldots \ldots$

Then problem $E_{\lambda}^{p^{\alpha}}$ has precisely three regular solutions.

And each time $\lambda=\mu_{n}$, there exists a secondary bifurcating continuum $\varepsilon_{n}$ of singular of non trivial solutions which are diffeomorphic to $[0, \pi]^{n}, n=1,2, \ldots$.

The following figures show how the bifurcating diagram corresponding to different cases studies above may look like:

First case : $p>2$ and $r(p, F)<1$

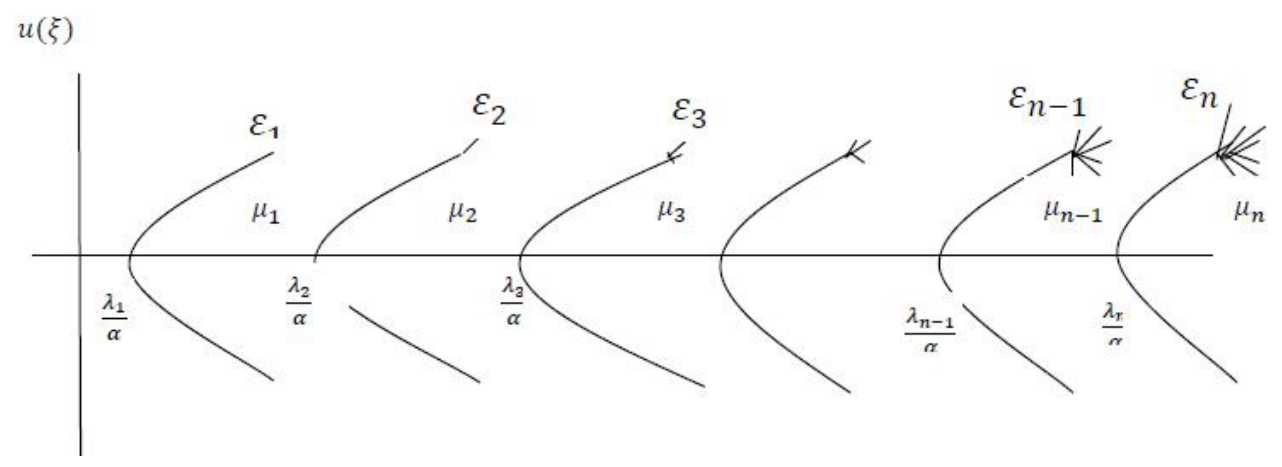

Figure 3: $u(\xi)=\max _{x \in] 0, \pi[}|u(x)|$ 
Second case: $p>2>$ and $r(p, F) \geq 1$



Figure 4: where []$=[r(p, F)], u(\xi)=\max _{x \in] 0, \pi[}|u(x)|$

We conclude this work by exhibiting an example which justifies our statements about the real number $r(p, F)$.

If we take $f(u)=-|u|^{2(p-1)} u+|u|^{p-2} u, p>2$, then we shall verify easily that $f$ satisfies all the hypotheses $\left.\left.\left.\left.\bar{H}_{1}\right), \bar{H}_{2}\right), \bar{H}_{3}\right), \bar{H}_{4}\right), \bar{H}_{5}, \bar{H}_{6}$, and $\left.i i i\right)$ and we find that:

$$
r(p, F)=\frac{\left(\Gamma\left(1-\frac{2}{p}\right)\right)^{2}}{2^{\frac{1}{p}} \Gamma\left(1-\frac{2}{p}\right)-\left(\Gamma\left(1-\frac{1}{p}\right)\right)^{2}}
$$

Where $\Gamma(x)=\int_{0}^{+\infty} t^{x-1} e^{x-1} e^{-t} d t$

The graph of $r(p, F)$ is as portrayed in figure 5

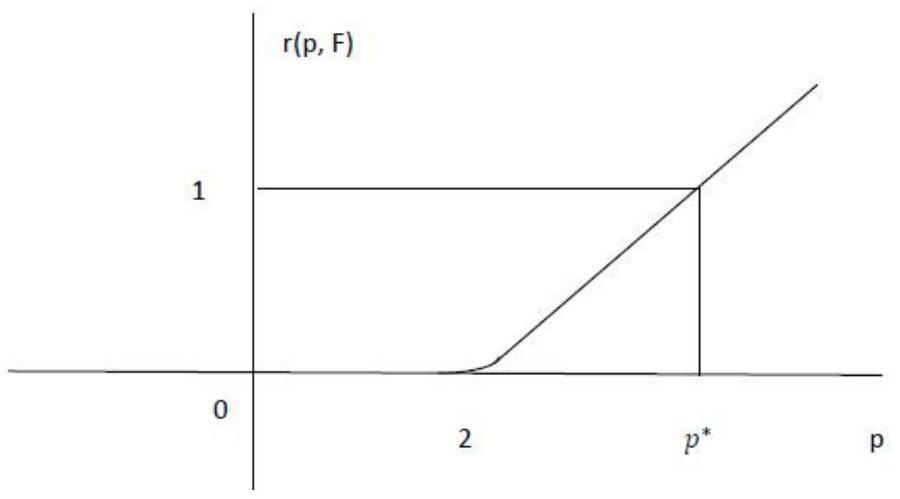

Figure 5: 
We notice that the real number $r(p, F)$ can take every possible positif value.

In fact, $p^{*}$ corresponds to the separation value of the two cases considered above; that is

$$
\begin{aligned}
& r(p, F)<1 \quad \forall p \in\left(1, p^{*}\right) \\
& r(p, F) \geq 1 \quad \forall p \geq p^{*}
\end{aligned}
$$

\section{Remark}

When $p^{*}>2$, there exists for some value of the parameter $\lambda$ (for example between $\mu_{1}$ and $\frac{\lambda_{2}}{\alpha}$ in theorem 3 ) what the physicists call a Dead core where the solution is necessarily constant, since between $\mu_{1}$ and $\frac{\lambda_{2}}{\alpha}$ the only non trivial solution is the singular one [2], [7].

\section{Study of the problem $E_{\lambda}^{p}$}

Let us now study the problem $E_{\lambda}^{p}$ in considerably more details. The main tool is the fixed point theorem.

Let us begin with the following definition

\section{Definition 1}

$(\lambda, u)$ is a solution of $E_{\lambda}^{p}$ if and only if $u$ and $\left|\frac{d u}{d x}\right|^{p-2} \frac{d u}{d x}$ are defined on $[0, \pi]$ and are of class $C^{1}$ on $[0, \pi]$ and satisfy (3) et (4).

\section{Remark 1}

One can also define the solutions of $E_{\lambda}^{p}$ in the weak sense:

$u$ is a solution if and only if:

$u \in W_{0}^{1, p}(0, \pi)$ and satisfies the equation $E_{\lambda}^{p}$ in the sense of distribution if and only if

$$
\int_{0}^{\pi}\left|\frac{d u}{d x}\right|^{p-2} \frac{d u}{d x} \cdot \frac{d v}{d x} d x=\lambda \int_{0}^{\pi} f(u) v d x \text { for all } v \in W_{0}^{1, p}(0, \pi)
$$

One can show that such solution is also a solution in the sense of the definition (1) above.

\section{Lemma 1}

i) If $a_{ \pm}$are both finite, then all the solutions $u(x)$ of $E_{\lambda}^{p}$ satisfy:

$$
a_{-} \leq u(x) \leq a_{+}
$$


ii) If $a_{ \pm}$are both infinite, then we have

$$
F(u(x)) \geq 0
$$

for all solutions of the problem $E_{\lambda}^{p}$.

\section{Proof}

i) Let $x_{0}$ be a point such that $u\left(x_{0}\right)=\operatorname{maxu}(x)$. In a such point, we have

$$
-\frac{d}{d x}\left(\left|\frac{d u}{d x}\right|^{p-2} \frac{d u}{d x}\left(x_{0}\right)\right) \geq 0
$$

Indeed, suppose the contrary, that is

$$
-\frac{d}{d x}\left(\left|\frac{d u}{d x}\right|^{p-2} \frac{d u}{d x}\left(x_{0}\right)\right)<0
$$

As $-\frac{d}{d x}\left(\left|\frac{d u}{d x}\right|^{p-2} \frac{d u}{d x}\right)$ is a continuous function, it follows that the inequality $(8)$ is still true in a neighborhood $\vartheta$ of $x_{0}$. This yields that $\left(\left|\frac{d u}{d x}\right|^{p-2} \frac{d u}{d x}\right)$ is strictly increasing for every $x \in \vartheta$, which in turn yields that $\frac{d u}{d x}$ is strictly increasing in $\vartheta$. And this contradicts the fact that $u$ possess a maximum in $x_{0}$. This yields $f\left(u\left(x_{0}\right)\right) \geq 0$, so that $u\left(x_{0}\right) \leq a_{+}$

In the same way, one can show that in a point $y_{0}$ such that $u\left(y_{0}\right)=$ $\min _{x} u(x)$ and one has $a_{-} \leq u\left(y_{0}\right)$. This together with the results above prove $i)$.

ii) It is an immediate consequence of the definition of F. It will be convenient below to write the problem $E_{\lambda}^{p}$ as a first order system. For that purpose, let us perform the change of variables:

$t=\lambda^{p} x$ in the equation (1) one obtains $E_{\lambda}^{p}$ :

$$
\begin{aligned}
& -\frac{d}{d t}\left(\left|\frac{d u}{d t}\right|^{p-2} \frac{d u}{d t}\right)=f(x) \\
& u(0)=u\left(\lambda^{\frac{1}{p}} \pi\right)=0
\end{aligned}
$$

Let us now consider now the following system $E_{\lambda}^{p}(\delta)$ :

$$
\begin{aligned}
& \frac{d u}{d t}=|w|^{p^{*}-2} w \\
& \frac{d w}{d t}=-f(u) \quad \frac{1}{p *}+\frac{1}{p}=1 \\
& u(0)=0, \quad w(0)=\delta
\end{aligned}
$$




\section{Lemma 2}

$(\mu, \lambda)$ is a solution of $E_{\lambda}^{p}$ if and only if there exists a $\delta$ such that $(u, w, \delta)$ is a solution of $E_{\lambda}^{p}(\delta)$ with $u\left(\lambda^{\frac{1}{p}} \pi\right)=0$.

\section{Proof}

Let $(u, \lambda)$ be a solution of $E_{\lambda}^{p}$, setting $w=\left|\frac{d u}{d t}\right|^{p-2} \frac{d u}{d t}$, it follows that $\frac{d u}{d t}=$ $|w|^{p *-2} w$, with $\frac{1}{p *}+\frac{1}{p}=1$, consequently $u$ and $w$ are of class $C^{1}$ and are solutions of the system $E_{\lambda}^{p}(\delta)$ with $u(\pi)=0$, then $(u, \lambda)$ is a solution of $E_{\lambda}^{p}$ with $w=\left|\frac{d u}{d t}\right|^{p-2} \frac{d u}{d t}$ and $u^{\prime}(0)=|\delta|^{p *-2} \delta$. We can can state

\section{Proposition 1}

For every $\delta$, the system $E_{\lambda}^{p}(\delta)$ has almost a solution $(u, w)$ defined entirely on $\mathbb{R}$.

\section{Proof}

It split in several steps.

\section{First step.}

The system $E_{\lambda}^{p}(\delta)$ can also be put in an integral equation form:

$$
\begin{gathered}
u(x)=\int_{0}^{x}|w|^{p^{*}-2} w(Z) d Z=T_{1}(w) \\
w(x)=\delta+\lambda \int_{0}^{x} f(u(Z)) d Z=T_{2}(u)
\end{gathered}
$$

$(u, w)$ is a solution of $E_{\lambda}^{p}(\delta)$ means that in $\mathbb{R},(u, w)$ satisfy (9) and (10) which, in turn, are equivalent to one of the following integral equations

$$
\begin{aligned}
u & =T_{1}\left(T_{2}(u)\right) \\
w & =T_{2}\left(T_{1}(w)\right)
\end{aligned}
$$

By taking into account the hypothesis $\bar{H}_{3}$, we show easily that for $x$ sufficiently small $T_{1} \circ T_{2}$ is a contraction on the Banach space $\mathcal{C}([0, x])$. And hence by fixed point theorem the equation (11) has one and only one local solution.

Consequently, for such an $x, T_{1} \circ T_{2}$ admits a fixed point in $\mathcal{C}([0, x])$. It is easily seen that this function $u$ is a solution of $E_{\lambda}^{p}(\delta)$ in $[0, x]$, with $u \in \mathcal{C}^{1}([0, x])$ and $\left|u^{\prime}\right|^{p^{*}-2} \quad u^{\prime} \in \mathcal{C}^{1}([0, x])$. Applying the general theory of first order system with continuous right hand, we can extend $u$ over a maximal interval [0,T] [5]. 


\section{Second step}

\section{Lemma 2}

Let $(u, w)$ be a solution of the system $E_{\lambda}^{p}(\delta)$, then we have $u \equiv 0, w \equiv 0$, or the zeros of $u$ and the zeros of $w$ are isolated.

\section{Proof}

Indeed, let $(u(t), w(t))$ be a non trivial solution of $E_{\lambda}^{p}(\delta), t_{0}$ be a zero of $u$ : $u\left(t_{0}\right)=0$. If we have $u^{\prime}\left(t_{0}\right)=0$, then $u$ would be a solution of the Cauchy problem:

$$
\begin{array}{rlrl}
\frac{d u}{d t} & =|w|^{p^{*}-2} w & & \\
\frac{d w}{d t} & =-f(u) & \frac{1}{p^{*}}+\frac{1}{p}=1 \\
u\left(t_{0}\right) & =0, \quad w\left(t_{0}\right)=0 &
\end{array}
$$

From above, we deduce that the only solution of this system is the trivial one. This contradiction shows that $u^{\prime}\left(t_{0}\right) \neq 0$ and hence the zeros of $u(t)$ are isolated. On the other hand, let $\bar{x}_{0}$ be such that $w\left(\bar{x}_{0}\right)=0$, from the first equation of $E_{\lambda}^{p}(\gamma)$, we get $u^{\prime}\left(\bar{x}_{0}\right)=0$.

If $u\left(\bar{x}_{0}\right)=0$, then one deduce as above that $u(t) \equiv 0$. Consequently $u^{\prime}\left(\bar{x}_{0}\right) \neq$ 0 and $w^{\prime}\left(\bar{x}_{0}\right)=-f\left(u\left(\bar{x}_{0}\right)\right)$, so two situations can occur.

i) $a_{-}<u\left(\bar{x}_{0}\right)<a_{+}$, then $w^{\prime}\left(\bar{x}_{0}\right) \neq 0$ and one deduce easily that the zeros of $w$ are isolated.

ii) If $u\left(\bar{x}_{0}\right)$ is equal to at least one of the numbers $a_{ \pm}$then $w\left(\bar{x}_{0}\right)=0$, consequently then zeros of $w$ may not be isolated.

Now let us consider now the following function

$$
\begin{aligned}
& V: \quad \mathbb{R}^{2} \quad \rightarrow \quad \mathbb{R} \\
& (u, w) \rightarrow V(u, w)
\end{aligned}
$$

Where $V(u, w)=\frac{1}{p^{*}}|w|^{p^{*}}+F(u)$ Let $(u(x), w(x))$ be a solution of $E_{\lambda}^{p}(\delta)$ and $V^{*}(x)=V(u(x), w(x))$, then we have.

\section{Third step.}

\section{Proposition}

$V^{*}$ is a first integral of the system $E_{\lambda}^{p}(\delta)$ :

$$
V^{*}(x)=E=\frac{1}{p^{*}}|\delta|^{p^{*}}
$$




\section{Proof}

Indeed, by multiplying the first equation of the system $E_{\lambda}^{p}(\delta)$ by $\frac{d w}{d t}$ and the second equation by $\frac{d u}{d t}$, we obtain:

$$
\begin{aligned}
& \frac{d u}{d t} \frac{d w}{d t}=|w|^{p^{*}-2} w \frac{d w}{d t} \\
& \frac{d w}{d t} \frac{d u}{d t}=-f(u) \frac{d u}{d t}
\end{aligned}
$$

From which one deduces by using the identity

$$
\begin{aligned}
& |u|^{p-2} u \frac{d u}{d t}=\frac{1}{p} \frac{d}{d t}\left(|u|^{p}\right) ; \quad p>1 \\
& \frac{d}{d t}\left(\frac{1}{p^{*}}|w|^{p^{*}}+F(u)\right)=0, \text { and hence } \\
& \frac{1}{p^{*}}|w|^{p^{*}}+F(u)=E=\frac{1}{p^{*}}|\delta|^{p^{*}}
\end{aligned}
$$

\section{Remark}

The existence of the first integral $V^{*}$ implies automatically then that solutions $(u, w)$ of $E_{\lambda}^{p}(\delta)$ are defined entirely on $\mathbb{R}$.

\section{The structure of the set of solutions of $E_{\lambda}^{p}(\delta)$.}

Now let us study the system $E_{\lambda}^{p}(\delta)$ in more details. The main tool is the phase plane. We interpret $E_{\lambda}^{p}(\delta)$ as a dynamical system defined on the phase plane $(u, w)$. The point of the trajectory has Cartesian coordinates $(u, v)$ and we call such point a representative point. We note that $u$ and $w$ are function of $t$, that is the curve in $(u, w)$ plane may be regarded as given in parametric form with $t$ as parameter. And the totality of all the solutions of $E_{\lambda}^{p}(\delta)$ may be described in the phase portrait of the system.

\section{Remark}

By analogy, the identity (3) expresses the law of conservation of energy; $\frac{1}{p^{*}}|w|^{p^{*}}$ represents the changes in kinetic energy, $F(u)$ represents the potential energy of the system $E_{\lambda}^{p}(\delta)$.

Now let us proceed to description of the energy levels of $E_{\lambda}^{p}(\delta)$ that is:

$$
\xi_{\delta}^{p}=\left\{(u, w): \frac{1}{p^{*}}|w|^{p^{*}}+F(u)=E=\frac{1}{p^{*}}|\delta|^{\frac{1}{p^{*}}}\right\}
$$


which is a continuously differentiable curve in the neighborhood of each one of its points, except the equilibrium position. In fact, we shall use the implicit function theorem: We have $\frac{\partial \xi_{\delta}^{p}}{\partial u}=f(u)$ or $\frac{\partial \xi_{\delta}^{p}}{\partial w}=(p-1)\left|\frac{d u}{d x}\right|^{p-2} \frac{d u}{d x}$ If one of these derivatives is different from zero in neighborhood of the considered point, then $\xi_{\delta}^{p}$ is represented by the graph of a differential curve of the form:

$$
u=G\left(u^{\prime}\right) \quad \text { or } \quad u^{\prime}=\bar{G}(u)
$$

Let $a_{ \pm}$be the singular points and set $F\left(a_{ \pm}\right)=E_{ \pm}$.

We propose to study the general aspect of the trajectory near the singular points.

One sees that for every a : $\frac{1}{p^{*}}|w|^{p^{*}}=E-F(u)$

so, every time the potential curve is slightly larger than the line $E, \frac{1}{p^{*}}|w|^{p^{*}}$ is negative, which is none sense, it follows that the representative point in the phase plane, never reaches those points where the curve $F(u)$ dominates the line $E$. In the common points of $F$ and the line $E$, the velocity of the system is equal zero.

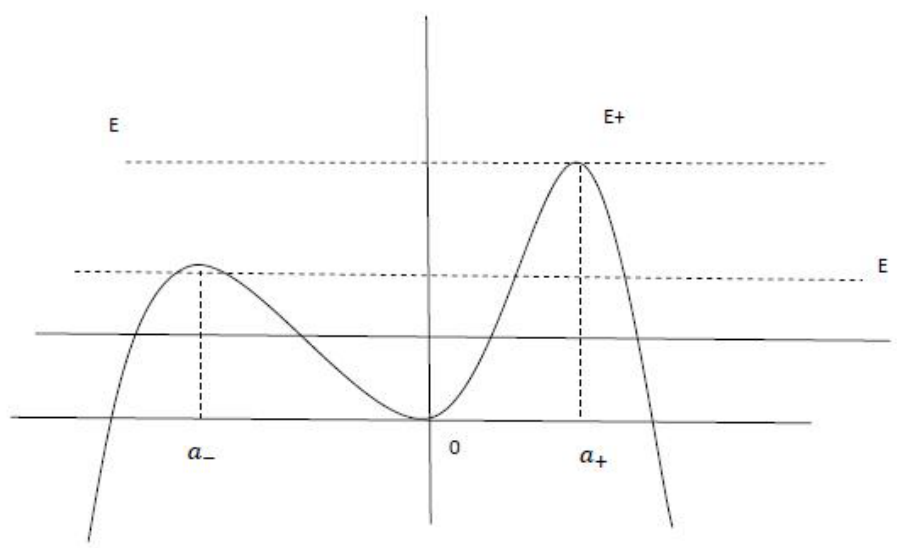

Figure 6:

\section{Inflexion Points}

Let a be a point such that

$$
\begin{aligned}
& E=F(a) \\
& \frac{d F}{d u}(a) \neq 0
\end{aligned}
$$






Figure 7:

Let $t_{0}$ such that

$$
u\left(t_{0}\right)=a
$$

Then $\frac{d u}{d t}\left(t_{0}\right)=0$, so the representation point never reaches the points which are larger than $a$. Then it must leaves the point of abscissa a and moves towards the left. So there exists $t_{1}>t_{0}$ such that.

$$
u\left(t_{1}\right)=b<a
$$

And

$$
E \neq F(u) \quad \forall u \in[b, a]
$$

On the other hand, as $\frac{d w}{d t}\left(t_{0}\right)=-f(a)<0$, it follows that the velocity at the point of abscissa $b$ is given by

$$
\left.\frac{d u}{d t}\right|_{t=t_{1}}=-\left\{\frac{p}{p-1}(E-F(u))\right\}^{\frac{1}{p}}
$$

The representative point reaches the point of abscissa $b$ at a time $t_{1}$ given by

$$
t_{1}-t_{0}=\left(\frac{p-1}{p}\right)^{\frac{1}{p}} \int_{b}^{a} \frac{d u}{(E-F(u))^{\frac{1}{p}}}
$$

We must check the convergence of this integral. By hypothesis, $F$ is at least twice continuously differentiable. So it may be expanded in Taylor series about the point a 


$$
\begin{aligned}
& F(u)=E+F^{\prime}(a)(u-a)+\frac{1}{2}(u-a)^{2} F^{\prime \prime}(a+\nu(u-a)) \quad 0<\nu<1 \\
& E-F(u)=(a-u)\left[F^{\prime}(a)+\frac{1}{2}(u-a) F^{\prime \prime}(a+\nu(u-a))\right]
\end{aligned}
$$

Let's put $\varphi(u)=F^{\prime}(u)+\frac{1}{2 !}(u-a) F^{\prime \prime}(a+\nu(u-a))$

We have $\varphi(u) \neq 0 \quad \forall u \in[a, b]$

Indeed $\varphi(a)=F(a) \neq 0$ because of (15)

And $\varphi(u) \neq 0 \quad \forall u \in[b, a]$, because on the contrary for such value of u, $E=F(a)$ which is impossible by (18).

So there exists a real number $k$ such that

$$
0<k<\varphi(u) \quad \forall u \in[b, a]
$$

Let's put

$$
I=\left(\frac{p-1}{p}\right)^{\frac{1}{p}} \int_{b}^{a}(E-F(u))^{\frac{1}{p}} d u=\left(\frac{p-1}{p}\right)^{-\frac{1}{p}} \int_{b}^{a}(a-u)^{-\frac{1}{p}}(\varphi(u))^{-\frac{1}{p}} d u
$$

But by virtue of (24), one has

$$
\begin{gathered}
(\phi(u))^{-\frac{1}{p}} \leq k^{-\frac{1}{p}} \quad \forall u \in[b, a] \quad \text { so } \\
I \leq k^{-\frac{1}{p}}\left(\frac{p-1}{p}\right)^{\frac{1}{p}} \int_{b}^{a}(a-u)^{-\frac{1}{p}} d u<+\infty
\end{gathered}
$$

The representative point reaches then the point of abscissa $b$ on a finite time. And going so on, one can show that, the trajectory corresponding to $E$, is closed in the phase plane. 


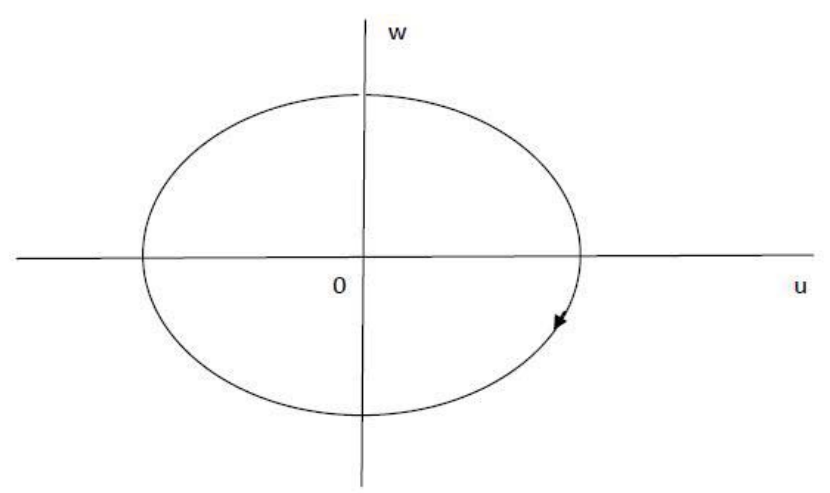

Figure 8:

\section{Stationary points}

Let $a_{+}$be the abscissa of a point where the curve of $F$ and the line $E$ are tangent:

$$
\begin{aligned}
& E_{+}=F\left(a_{+}\right) \\
& \frac{d F}{d u}\left(a_{+}\right)=0
\end{aligned}
$$

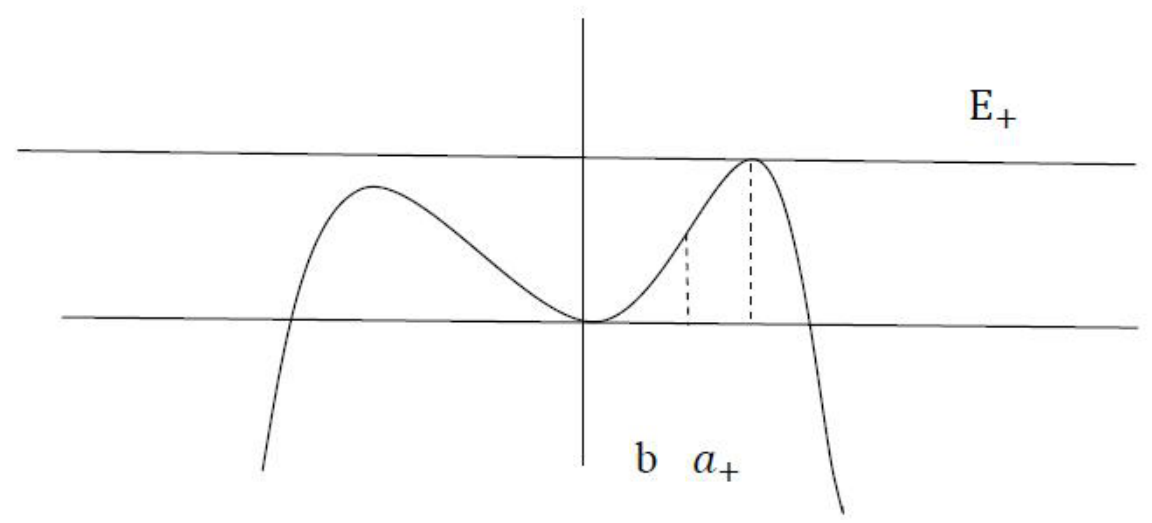

Figure 9:

Let $t_{0}$ be such that:

$$
u\left(t_{0}\right)=a_{+}
$$


We suppose that the line $E_{+}$dominates the trajectory on some interval on the left of $a_{+}$:

$\exists b<a_{+}$such that

$$
E_{+}>F(u) \quad \forall u \in\left[b, a_{+}[\right.
$$

We also suppose that

$$
u\left(t^{*}\right)=b \text { and } \frac{d u}{d t}\left(t^{*}\right)>0
$$

One can write

$$
F(u)=E+\frac{1}{2 !}(u-a)^{2} F^{\prime \prime}\left(a_{+}+\theta\left(u-a_{+}\right)\right)
$$

Let set

$$
\varphi(u)=-F^{\prime \prime}\left(u+\theta\left(u-a_{+}\right)\right)
$$

By virtue of (28) one has

$$
\varphi(u)>0 \quad \forall\left[b, a_{+}[\right.
$$

The function $\varphi$ is continuous on $\left[b, a_{+}\right]$, so it is bounded:

$$
\varphi(u) \leq K \quad \forall u \in\left[b, a_{+}\right]
$$

For $b \leq \alpha<a_{+}$let $I_{\alpha}$ be defined as follows

$$
\begin{aligned}
I_{p} & =\left(\frac{p-1}{p}\right)^{\frac{1}{p}} \int_{0}^{\alpha}\{E-F(u)\}^{-\frac{1}{p}} d u \\
& =2^{\frac{1}{p}}\left(\frac{p-1}{p}\right)^{\frac{1}{p}} \int_{0}^{\alpha}\left(a_{+}-u\right)^{-\frac{2}{p}}(\varphi(u))^{-\frac{2}{p}} d u
\end{aligned}
$$

In term of (30) one has $I_{\alpha} \geq 2^{\frac{1}{p}}\left(\frac{p-1}{p}\right)^{\frac{1}{p}} K^{-\frac{2}{p}} \int_{0}^{p}\left(a_{+}-u\right)^{-\frac{2}{p}} d u$

Two cases should be considered:

First case A: $p \leq 2$

We obtain immediately from above that

$$
\lim _{a \rightarrow a_{+}} I_{\alpha}=+\infty
$$

That is the representation point reaches the point of abscissa $a_{+}$in an infinite time. 
Second case B: $p>2$

The convergence or the divergence of the integral above depends on the aspect of the function $F$ in the neighborhood of the point $a_{+}$. That is two situations can occur:

$$
\begin{aligned}
& \left(\frac{p-1}{p}\right)^{\frac{1}{p}} \int_{0}^{a_{+}}\left\{F\left(a_{+}\right)-F(u)\right\}^{-\frac{1}{p}} d u=+\infty \\
& \left(\frac{p-1}{p}\right)^{\frac{1}{p}} \int_{0}^{+\infty}\left\{F\left(a_{+}\right)-F(u)\right\}^{-\frac{1}{p}} d u<+\infty
\end{aligned}
$$

Finally, there are two cases which we shall suppose depending on whether $a_{ \pm}$are either finite or infinite.

i- $a_{ \pm}$are both finite.

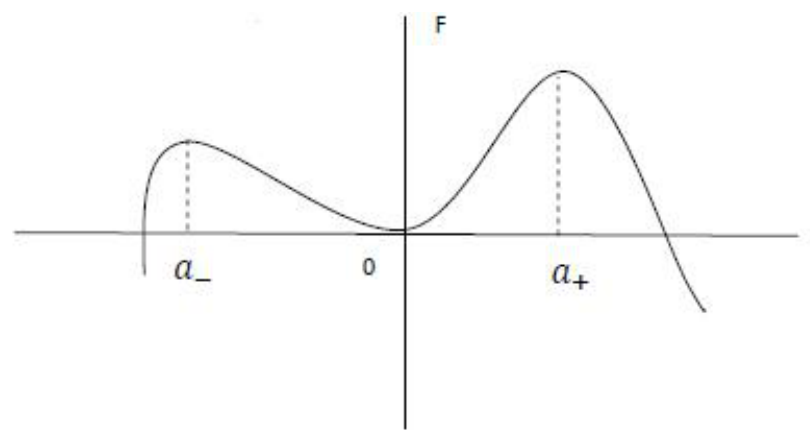

Figure 10:

In this case the portrait phase looks like

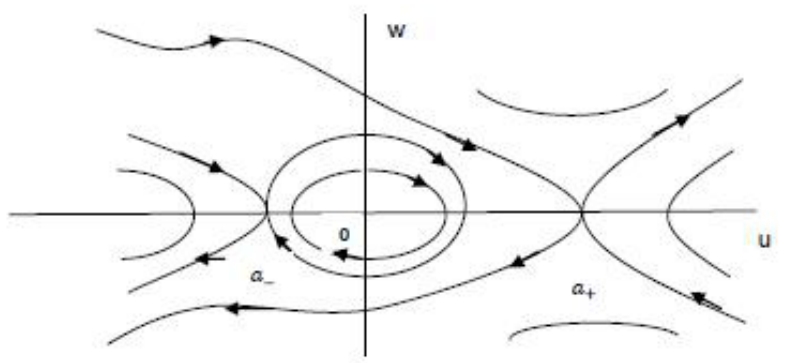

Figure 11: 
ii) $a_{ \pm}$are both infinite.

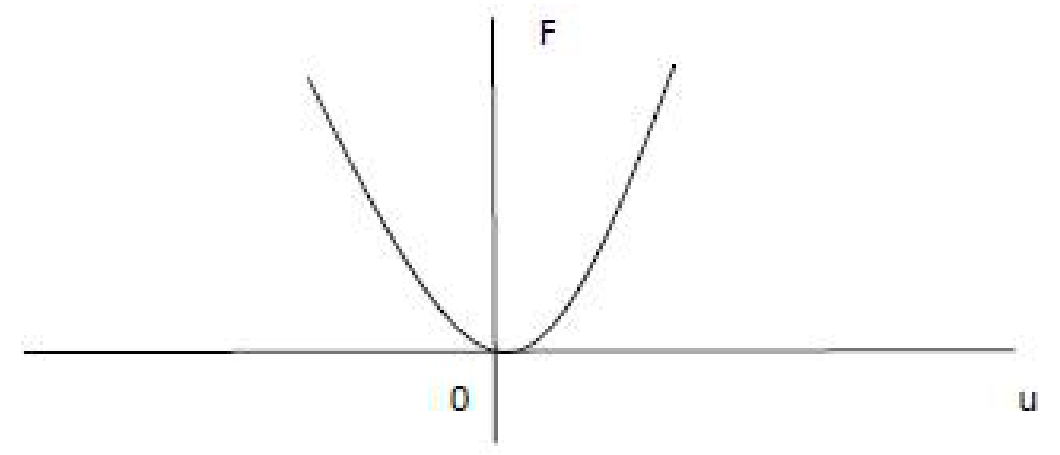

Figure 12:

In this case, the portrait phase looks like

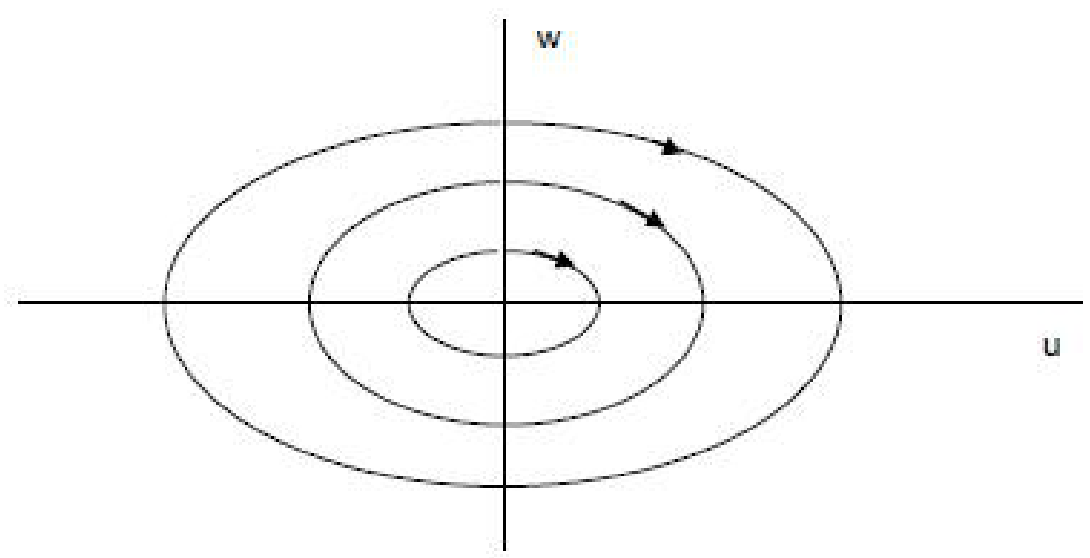

Figure 13:

\section{Bifurcations equations associated with $E_{\lambda}^{p}(\delta)$}

Let us recall the main hypotheses made on the nonlinearity $f$. For this, let $F: \mathbb{R} \rightarrow \mathbb{R}$ be the function defined by 


$$
F(u)=\int_{0}^{u} f(\xi) d \xi, \quad u \in \mathbb{R}
$$

We suppose that there exists unique numbers $a_{-}$and $a_{+}$such that:

$$
\begin{gathered}
-\infty \leq a_{-}<0<a_{+} \leq+\infty \\
\operatorname{sgn} f(u)=\operatorname{sgn} u, \quad a_{-}<u<a_{+}
\end{gathered}
$$

And either $a_{+}=+\infty$ or $f\left(a_{+}\right)=0$ (resp. $a_{-}=-\infty$ or $f\left(a_{-}\right)=0$ )

Further, we suppose that outside the interval $\left(a_{-}, a_{+}\right)$, one has:

$$
\left.\bar{H}_{6}\right): f(u) u<0
$$

We introduce two quantities $E_{ \pm}$, through:

$$
E_{ \pm}=\lim _{u \rightarrow a_{ \pm}} F(u)
$$

We note that $F$ is strictly increasing on $\left[0, a_{+}\right)$and strictly decreasing on $\left(a_{-}, 0\right]$. So on $\left[0, a_{+}\right)$, the function $F$ has an inverse

$$
m_{+}:\left[0, E_{+}\right) \rightarrow\left[0, a_{+}\right)
$$

And on $\left(a_{+}, 0\right]$, the function $F$ has an inverse

$$
m_{-}:\left[0, E_{-}\right) \rightarrow\left(a_{-}, 0\right]
$$

We define two functions $l_{ \pm}(E)$ in the following way:

$$
\begin{aligned}
& l_{+}(E)=\left(\frac{p-1}{p}\right)^{\frac{1}{p}} \int_{0}^{m_{+}(E)}\{E-F(u)\}^{-\frac{1}{p}} d u, \quad 0<E<E_{+} \\
& l_{-}(E)=\left(\frac{p-1}{p}\right)^{\frac{1}{p}} \int_{m_{-}(E)}^{0}\{E-F(u)\}^{-\frac{1}{p}} d u, \quad 0<E<E_{-}
\end{aligned}
$$

More precisely, the significance of $l_{ \pm}(E)$ is as follows. Let us consider a trajectory on the phase plane corresponding to a given constant $E$ : $a_{+}$are both finite 




Figure 14:

$a_{ \pm}$are both infinite



Figure 15:

It follows from $\S I I l_{+}(E)$ (resp. $\left.l_{-}(E)\right)$ is the time required for the representative point $(u(t), v(t))$ to traverse one loop in $u>0$ (resp. $u<0$ ).

Consider $l_{+}(E)$ for given $E, 0<E<E_{+}$. We make the change of variables

$$
E y^{p}=F(u) \quad 0 \leq y \leq 1, \quad 0 \leq u \leq m_{+}(E)
$$

This leads us to the relation: 


$$
\begin{aligned}
l_{+}(E) & =p\left(\frac{p-1}{p}\right)^{\frac{1}{p}} E^{\frac{p-1}{p}} \int_{0}^{1}\left\{1-y^{p}\right\}^{-\frac{1}{p}} \frac{y^{p-1}}{f(u)} d y \\
u & =m_{+}\left(E y^{p}\right), \quad 0 \leq y \leq 1,0<E<E_{+}
\end{aligned}
$$

Similarly, if one put: $E y^{p}=F(u) 0 \leq y \leq 1, \quad 0 \leq u \leq m_{-}(E)$, we obtain

$$
\begin{aligned}
& l_{-}(E)=p\left(\frac{p-1}{p}\right)^{\frac{1}{p}} E^{\frac{p-1}{p}} \int_{-1}^{0}\left\{1-y^{p}\right\}^{-\frac{1}{p}} \frac{y^{p-1}}{f(u)} d y \\
& u=m_{-}\left(E y^{p}\right), \quad 0 \leq y \leq 1,0<E<E_{-}
\end{aligned}
$$

We can prove:

\section{Proposition 3}

The functions $l_{ \pm}(E)$ are continuous on $\left(0, E_{ \pm}\right)$. Furthermore

$$
\lim _{E \rightarrow 0^{+}} l_{+}(E)=\frac{1}{p}\left(\frac{p-1}{\alpha}\right)^{\frac{1}{p}} B\left(\frac{1}{p}, 1-\frac{1}{p}\right)
$$

Where $\alpha$ is given by $\bar{H}_{3}$ and $B(x, y)$ is the beta function.

\section{Proof}

From $\bar{H}_{3}$ it follows that:

$$
\forall \varepsilon>0,0<\varepsilon<1, \exists \delta>0 \text { such that }
$$

If $0 \leq u \leq \delta$, then

$$
\alpha(1-\varepsilon) u^{p-1} \leq f(u) \leq \alpha(1+\varepsilon) u^{p-1}
$$

Given this $\delta$, we can find $\eta$ with $0<\eta<E_{+}$such that if, $0 \leq E \leq \eta$, then $m_{+}(E) \leq \delta$. This follows from continuity of $m_{+}(E)$.

Now, we have by integrating (40):

$$
\frac{\alpha}{p}(1-\varepsilon) u^{p} \leq F(u) \leq \frac{\alpha}{p}(1+\varepsilon) u^{p}, \quad 0 \leq u \leq \delta
$$

Hence elevating it to the power $\frac{1}{p}$ :

$$
\left(\frac{\alpha}{p}(1-\varepsilon)\right)^{\frac{1}{p}} u \leq(F(u))^{\frac{1}{p}} \leq\left(\frac{\alpha}{p}(1+\varepsilon)\right)^{\frac{1}{p}} u
$$


Therefore as

$$
E y^{p}=F(u), 0 \leq y \leq 1, \quad 0 \leq u \leq m_{+}(E)
$$

one obtains:

$$
\left(\frac{\alpha(1-\varepsilon)}{p E}\right)^{\frac{p-1}{p}} u^{p-1} \leq y^{p-1} \leq\left(\frac{\alpha(1+\varepsilon}{p E}\right)^{\frac{p-1}{p}} u^{p-1}
$$

With $0 \leq E \leq \eta, 0 \leq y \leq 1$. From (40) one has:

$$
\frac{(\alpha(1-\varepsilon))^{-1}}{u^{p-1}} \leq \frac{1}{f(u)} \leq \frac{(\alpha(1+\varepsilon))^{-1}}{u^{p-1}}
$$

Hence

$$
\left(\frac{\alpha(1-\varepsilon)}{p E}\right)^{\frac{p-1}{p}} \frac{1}{\alpha(1+\varepsilon)} \leq \frac{y^{p-1}}{f(u)} \leq\left(\frac{\alpha(1+\varepsilon)}{p E}\right)^{\frac{p-1}{p}} \frac{1}{\alpha(1-\varepsilon)}
$$

With $0 \leq E \leq \eta, 0 \leq y \leq 1$.

From this and the definition of $l_{+}(E)$, there follows:

$$
(p-1)^{\frac{1}{p}} \frac{(1-\varepsilon)^{\frac{p-1}{p}}}{\alpha^{\frac{1}{p}}(1+\varepsilon)} \int_{0}^{1}\left\{1-y^{p}\right\}^{-\frac{1}{p}} d y \leq l_{+}(E) \leq \frac{(1+\varepsilon)^{\frac{p-1}{p}}}{\alpha^{\frac{1}{p}}(1-\varepsilon)} \int_{0}^{1}\left(1-y^{p}\right)^{-\frac{1}{p}} d y
$$

By taking into account that $\int_{0}^{1}\left\{1-y^{p}\right\}^{-\frac{1}{p}} d y=\frac{1}{p} B\left(\frac{1}{p}, 1-\frac{1}{p}\right)$, we obtain:

$$
\lim _{E \rightarrow 0^{+}} l_{+}(E)=\frac{1}{p}\left(\frac{p-1}{\alpha}\right)^{\frac{1}{p}} B\left(\frac{1}{p}, 1-\frac{1}{p}\right)
$$

In an entirely similar manner, one obtains

$$
\lim _{E \rightarrow 0^{+}} l_{-}(E)=\frac{1}{p}\left(\frac{p-1}{\alpha}\right)^{\frac{1}{p}} B\left(\frac{1}{p}, 1-\frac{1}{p}\right)
$$

Let us now investigate the behavior of $l_{ \pm}(E)$ when $E$ tends to $E_{ \pm}$. For this, we shall distinguish several cases:

$$
A: 1<p \leq 2
$$

In this case, we make no more hypotheses on $f$ outside $\left.\bar{H}_{1}\right) \ldots \bar{H}_{6}$ ).

$$
B: p>2
$$

In this case, we shall distinguish the following sub-cases: 
i. $a_{ \pm}$are both infinite;

ii. $a_{ \pm}$are both finite, and the two following integrals are infinite:

$$
\begin{aligned}
& \int_{0}^{a_{+}}\left\{F\left(a_{+}\right)-F(u)\right\}^{\frac{1}{p}} d u=+\infty \\
& \int_{a_{-}}^{0}\left\{F\left(a_{-}\right)-F(u)\right\}^{-\frac{1}{p}} d u=+\infty
\end{aligned}
$$

iii. $a_{ \pm}$are both infinite and the two following integrals are finite:

$$
\begin{gathered}
\int_{0}^{a^{+}}\left\{F\left(a_{+}\right)-F(u)\right\}^{-\frac{1}{p}} d u<+\infty \\
\int_{a_{-}}^{0}\left\{F\left(a_{-}\right)-F(u)\right\}^{-\frac{1}{p}} d u<+\infty
\end{gathered}
$$

Then we have:

\section{Proposition 4}

1. Under the statements $A$ or $B, i)$, ii), one has:

$$
\lim _{E \rightarrow E_{ \pm}} l_{ \pm}(E)=+\infty
$$

2. Under the statements $B$, iii), one has

$$
\begin{aligned}
& \lim _{E \rightarrow E_{+}} l_{+}(E)=\left(\frac{p-1}{p}\right)^{\frac{1}{p}} \int_{0}^{a_{+}}\left\{E_{+}-F(u)\right\}^{-\frac{1}{p}} d u<+\infty \\
& \lim _{E \rightarrow E_{-}} l_{-}(E)=\left(\frac{p-1}{p}\right)^{\frac{1}{p}} \int_{a_{-}}^{0}\left\{E_{-}-F(u)\right\}^{-\frac{1}{p}} d u<+\infty
\end{aligned}
$$

\section{Proof}

1. We render that proof only for $l_{+}(E)$, the proof for $l_{-}(E)$ is similar. For this, firstly let's suppose that $a_{+}<+\infty$, then under the condition $A$, an entirely similar argument like the one made in $\S I$ I shows that

$$
\lim _{E \rightarrow E_{ \pm}} l_{ \pm}(E)=+\infty
$$

While that under the statements $B, i)$, ii), the result is an immediate consequence of the hypotheses (45) et (46). 
Now suppose that $a_{+}=+\infty$, then by virtue of hypotheses made on $f, f$ is strictly positive for $u \in(0,+\infty)$ and Hence by $\bar{H}_{3}$ :

$$
\lim _{u \rightarrow+\infty} \frac{f(u)}{u^{p-1}}=0
$$

Therefore,

$$
\lim _{u \rightarrow+\infty} \frac{F(u)}{u^{p}}=\lim _{u \rightarrow+\infty} \frac{f(u)}{p u^{p-1}}=0
$$

Let $\xi=\sup \left\{\frac{f(u)}{u^{p-1}}, 0<u<+\infty\right\}>0$. Choose any $\varepsilon$ sufficiently small with $0<\varepsilon<\xi$, then there exists an $u_{0} \in(0,+\infty)$ such that:

$f\left(u_{0}\right)=\varepsilon u_{0}^{p-1}$ and $f(u) \geq \varepsilon u^{p-1}$ for $u$ satisfying

$$
0 \leq u \leq u_{0}
$$

Hence

$$
F(u) \geq \frac{\varepsilon}{p} u^{p}, \quad \text { for } u \in\left[0, u_{0}\right]
$$

This together with (53) imply that there exists a $\bar{u} \in\left[u_{0},+\infty\right)$ such that

$$
F(u) \leq \frac{\varepsilon}{p} u^{p}, \quad \text { for every } u \in[0, \bar{u}]
$$

and

$$
F(\bar{u})=\frac{\varepsilon}{p} u^{p}=\bar{E}
$$

referring to (34), we see that

$$
\begin{aligned}
l_{ \pm} & =\int_{0}^{\bar{u}}\{\bar{E}-F(u)\}^{-\frac{1}{p}} d u \\
& \geq \int_{0}^{\bar{u}}\left\{\frac{\varepsilon}{p} \bar{u}^{p}-\frac{\varepsilon}{p} u^{p}\right\}^{-\frac{1}{p}} d u
\end{aligned}
$$

and after some computation, one obtains

$$
l_{ \pm}(\bar{E}) \geq \frac{1}{p}\left(\frac{p}{\varepsilon}\right)^{\frac{1}{p}} B\left(\frac{1}{p}, 1-\frac{1}{p}\right)
$$

That is $l_{ \pm} \rightarrow+\infty$, when $E \rightarrow E_{+}$.

2. The result is immediate by virtue of (50) and (51) 


\section{Proposition 5}

The function $l_{+}(E)$ are differentiable in their domain $\left(0, E_{ \pm}\right)$and are stricly increasing in $\left(0, E_{ \pm}\right)$.

\section{Proof}

We render it only for $l_{+}(E)$.

One has.

$$
\frac{d l_{+}}{d E}=\left(\frac{p-1}{p}\right)^{\frac{1}{p}} E^{-\frac{1}{p}} \int_{0}^{1}\left\{1-y^{p}\right\}^{-\frac{1}{p}} \frac{y^{p-1}}{f(u)}\left[\frac{(p-1) f^{2}(u)-p f(u) F^{\prime}(u)}{f^{2}(u)}\right] d y
$$

where $E y^{p}=F(u), \quad 0<E<E_{+}, \quad 0 \leq y \leq 1$

Let's define a function $\Im: \mathbb{R} \rightarrow \mathbb{R}$ by setting

$$
\begin{array}{ccc}
\Im(u)=(p-1) f^{2}(u)-p f^{\prime}(u) F(u) \\
F(u)>0 & \Leftrightarrow \quad p\left(\frac{f^{2}(u)-f^{\prime}(u) F(u)}{f^{2}(u)}\right)>1 \\
& \Leftrightarrow & \left(\frac{F(u)}{f(u)}\right)^{\prime}>\frac{1}{p}
\end{array}
$$

Which is the case by virtue of $\bar{H}_{5}$.

And one finds readily that the corresponding phase portrait looks like:

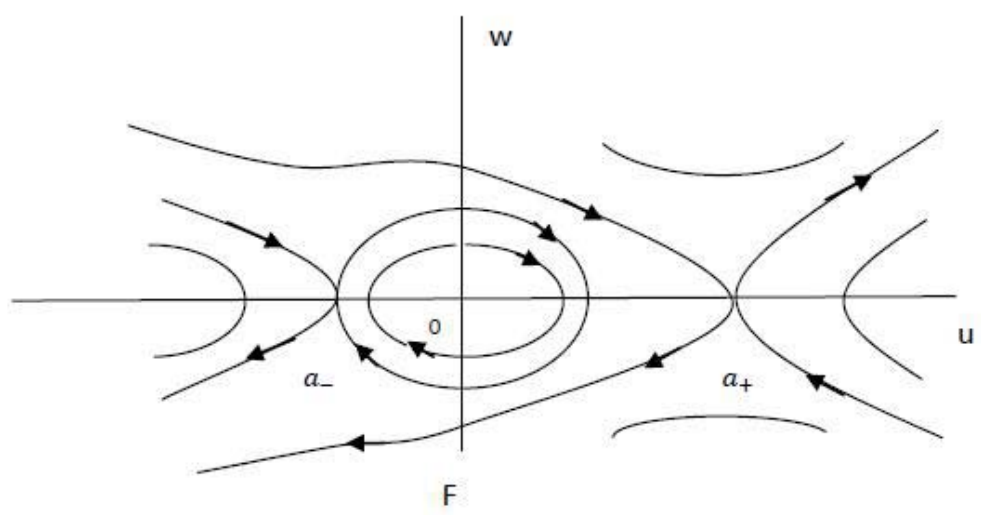

Figure 16: 
It follows from the above study, that under the case $p>2$ and $a_{ \pm}$are both finite, two possibilities can occur relatively to the heteroclinic orbits. « That is the curves which pass through the single points $\left(a_{-}, 0\right)$ and $\left(a_{+}, 0\right) \gg$; either one can traverse the heteroclinic orbit in an infinite time or in a finite time, the later case leads us to consider two kinds of solutions.

\section{Definition 1}

By a regular solution of problem $E_{\lambda}^{p}$, we mean every solution $u$ which has a finite number of zeros in $(0, \pi)$ and whose first derivative vanishes only once between two consecutive zeros.

\section{Definition 2}

By a singular solution of problem $E_{\lambda}^{p}$, we mean every solution $u$ which has a finite number of zeros in $(0, \pi)$, and such that between two consecutive zeros, the first derivative vanishes at least in a subinterval.

Geometrically speaking, a singular solution corresponds to a heteroclinic orbit for which the representation point $(u(t), w(t))$ puts a finite time $l_{+}\left(E_{+}\right)$(resp $\left.l_{-}\left(E_{-}\right)\right)$to reach the point $\left(a_{+}, 0\right)\left(\operatorname{resp}\left(a_{-}, 0\right)\right)$; and then makes a pose at point $\left(a_{+}, 0\right)\left(\operatorname{resp}\left(a_{-}, 0\right)\right)$; and continues on its path.

From this, the claims about the number and disposition of solutions follow. More precisely. If $\frac{p-1}{p}\left|\frac{d u}{d x}\right|^{p}+F(u)=E$, then the equation $E_{\lambda}^{p}(\delta)$ admits a regular solution $u$ satisfying $u\left(\lambda^{\frac{1}{p}} \pi\right)=0$ If and only if there exists an integer number $n$ and a constant $E$ such that one of the following bifurcation equations is satisfied

$$
\begin{aligned}
& 2 n l_{+}(E)+2(n-1) l_{-}(E)=\lambda^{\frac{1}{p}} \pi \\
& 2(n-1) l_{+}(E)+2 n l_{-}(E)=\lambda^{\frac{1}{p}} \pi \\
& 2 n l_{+}(E)+2 n l_{-}(E)=\lambda^{\frac{1}{p}} \pi \quad n=1,2, \ldots
\end{aligned}
$$

Furthermore, one must of course include the singular solutions which appear in the case where $E=F\left(a_{+}\right)\left(\operatorname{resp} E=F\left(a_{-}\right)\right)$. We shall return to them later in more details

Before discussing in more details the bifurcation equations, we shall add other hypotheses on $f$ in thecase $B, i i i)$, namely:

$$
\left.\bar{H}_{7}\right): \quad f(-u)=-f(u) ; \quad \forall u \in \mathbb{R}
$$

And let us consider the following real number whose significance only appears later: 


$$
r(p, F)=\frac{1}{p^{\frac{p-1}{p}} \alpha^{\frac{1}{p}} \frac{T(F)}{B\left(\frac{1}{p}, 1-\frac{1}{p}\right)}-1}
$$

where

$$
T(F)=\int_{0}^{\bar{a}}\{F(a)-F(u)\}^{-\frac{1}{p}} d u ; a=-a_{-}=a_{+}
$$

Let us now study the bifurcation equations. For this, we have:

\section{Proposition 6}

Let $\bar{\lambda}_{n}=\frac{\lambda_{n}}{\alpha} ; \quad n=1,2, .$.

where $\lambda_{n}$ is the eigenvalue of the problem $\wp(\lambda)$ and $\alpha$ is given by $\left.\bar{H}_{3}\right)$, then we have:

I) If one of the statements $A$ or $B, i)$, ii) holds, then, for each $n \geq 1$, there exist two function $E_{n}^{ \pm}$continuously mapping $\left[\bar{\lambda}_{n},+\infty[\right.$ into $[0, E[$ which have the following properties:

a) $\forall n \geq 1, \forall \lambda \in\left[\bar{\lambda}_{2 n-1},+\infty\left[, E_{2 n-1}^{+}(\lambda)\right.\right.$ (resp $\left.E_{2 n-1}^{-}(\lambda)\right)$ is the unique solution of the equation $(58)(\operatorname{resp}(59))$.

b) $\forall n \geq 1, \quad \forall \lambda \in\left[\bar{\lambda}_{2 n},+\infty\left[\right.\right.$ we have $E_{2 n}^{-}(\lambda)=E_{2 n}^{+}(\lambda)$ and $E_{2 n}^{+}(\lambda)$ is the unique solution of the equation (60).

II) Under the statement $B$, iii), we shall distinguish two sub cases: depending on whether $r(p, F)<1$ or $r(p, F) \geq 1$.

First sub case: $r(p, F)<1$

Then we have:

For every $n \geq 1$, there exists two functions $E_{n}^{ \pm}$continuously mapping $\left[\bar{\lambda}_{n}, \mu_{n}\right]$ into $[0, \bar{E}]$ which have the following properties:

a) $\forall n \geq 1, \forall \lambda \in\left[\bar{\lambda}_{2 n-1}, \mu_{2 n-1}\right] ; E_{2 n-1}^{+}(\lambda)$ (resp. $\left.E_{2 n-1}^{-}(\lambda)\right)$ is the unique solution of the equation (58) (resp (59)).

b) $\forall n \geq 1, \forall \lambda \in\left[\bar{\lambda}_{2 n}, \mu_{2 n}\right]$ we have $E_{2 n}^{-}=E_{2 n}^{+}(\lambda)$ and $E_{2 n}^{-}(\lambda)$ is the unique solution of the equation (60)

Second sub-case: $r(p, F) \geq 1$

Then we have:

For every $n$ such that: $1 \leq n \leq[r(p, F)]$; there exist two functions $E_{2 n-1}^{ \pm}(\lambda)$ continuously mapping $\left[\bar{\lambda}_{n}, \mu_{n}\right]$ into $[0, \bar{E}]$ which have the following properties. 
a) $\forall n \in[1,[r(p, F)]], \forall \lambda \in\left[\bar{\lambda}_{2 n-1}, \mu_{2 n-1}\right], E_{2 n-1}^{+}(\lambda)\left(\operatorname{resp} . \quad E_{2 n-1}^{-}(\lambda)\right)$ is the unique solution of the equation (58)(resp (59)).

b) $\forall N \in[1,[r(p, F)]], \forall \lambda \in\left[\bar{\lambda}_{2 n}, \mu_{2 n}\right]$, we have $E_{2 n}^{-}(\lambda)=E_{2 n}^{+}(\lambda)$ and $E_{2 n}^{+}(\lambda)$ is the unique solution of the equation (60).

Furthermore, for every $\lambda \in\left(\mu_{2 n-1}, \bar{\lambda}_{2 n-1}\right]$ (resp. $\left.\forall \lambda \in\left(\mu_{2 n}, \bar{\lambda}_{2(n+1)}\right]\right)$ zero is the only unique solution of the equations $(58),(59)$ et $(60)$

\section{Finally:}

$\forall n \geq[r(p, F)]+1$, there exist two functions $E_{n}^{ \pm}$continuously mapping $\left[\bar{\lambda}_{n}, \mu_{n}\right]$ into $[0, \bar{E}]$ which have the following properties:

a- $\forall n \geq[r(p, F)]+1, \forall \lambda \in\left[\bar{\lambda}_{2 n-1}, \mu_{2 n-1}\right], E_{2 n-1(\lambda)}^{+}\left(\operatorname{resp} . \quad E_{2 n-1}^{-}(\lambda)\right)$ is the unique solution of the equation (58) (resp. (59)).

b- $\forall n \geq[r(p, F)]+1, \forall \lambda \in\left[\bar{\lambda}_{2 n}, \mu_{2 n}\right]$, we have $E_{2 n}^{-}(\lambda)=E_{2 n}^{+}(\lambda)$ and $E_{2 n}^{+}(\lambda)$ is the unique solution of the equation (60) where

- $[x]$ is the integer part of $x$,

- $E^{-}=E_{+}=E_{-}=F(a)$,

$$
\mu_{n}=\frac{2^{p} n^{p}(p-1)}{\pi^{p} p}\left(\int_{0}^{a}\{F(a)-F(u)\}^{-\frac{1}{p}} d u\right)^{p}
$$

\section{Proof}

\section{Setting}

$$
\begin{aligned}
& l_{+}(E)=\left(\frac{p-1}{p}\right)^{\frac{1}{p}} T_{+}(E) \\
& l_{-}(E)=\left(\frac{p-1}{p}\right)^{\frac{1}{p}} T_{-}(E)
\end{aligned}
$$

Where

$$
\begin{aligned}
& l_{+}(E)=\int_{O}^{m_{+}(E)}\{E-F(u)\}^{-\frac{1}{p}} d u \\
& l_{-}(E)=\int_{m_{-}(E)}^{0}\{E-F(u)\}^{-\frac{1}{p}} d u
\end{aligned}
$$


Then, one can write the equations (58), (59) and (60) in the following manner:

$$
\begin{aligned}
& \frac{2 n(p-1)^{\frac{1}{p}}}{p^{\frac{1}{p}} \pi} T_{+}(E)+\frac{2(n-1)(p-1)^{\frac{1}{p}}}{p^{\frac{1}{p}}} T_{-}(E)=\lambda^{\frac{1}{p}} \\
& \frac{2(n-1)(p-1)^{\frac{1}{p}}}{p^{\frac{1}{p}} \pi} T_{+}(E)+\frac{2 n(p-1)^{\frac{1}{p}}}{p^{\frac{1}{p}} \pi} T_{-}(E)=\lambda^{\frac{1}{p}} \\
& \frac{2 n(p-1)^{\frac{1}{p}}}{p^{\frac{1}{p}} \pi} T_{+}(E)+\frac{2 n(p-1)^{\frac{1}{p}}}{p^{\frac{1}{p}} \pi} T_{-}(E)=\lambda^{\frac{1}{p}}
\end{aligned}
$$

It follows from propositions $(3 . \S I I I)$ and $(4 . \S I I I)$ that these three equations are defined only on the interval $\left(0, \min \left(E_{-}, E_{+}\right)\right)$. Let us now consider the equation (64) (resp (65)). Setting

$$
\begin{aligned}
& \psi_{2 n-1}^{+}(E)=\frac{2 n(p-1)^{\frac{1}{p}}}{p^{\frac{1}{p}} \pi} T_{+}(E)+\frac{2(n-1)(p-1)^{\frac{1}{p}}}{p^{\frac{1}{p}} \pi} T_{-}(E) \\
& \psi_{2 n-1}^{-}(E)=\frac{2(n-1)(p-1)^{\frac{1}{p}}}{p^{\frac{1}{p}} \pi} T_{+}(E)+\frac{2 n(p-1)^{\frac{1}{p}}}{p^{\frac{1}{p}} \pi} T_{-}(E)
\end{aligned}
$$

Then one has by propositions (3§III) and (4.§III) that:

$$
\begin{aligned}
& \lim _{E \rightarrow 0_{+}} \psi_{2 n-1}^{+}(E)=\bar{\lambda}_{2(2 n-1)}^{\frac{1}{p}} \\
& \lim _{E \rightarrow 0_{+}} \psi_{2 n-1}^{-}(E)=\bar{\lambda}_{2(2 n-1)}^{\frac{1}{p}}
\end{aligned}
$$

Using proposition $(4 \S I I I)$, we have

$$
\lim _{E \rightarrow \min \left(E_{-}, E_{+}\right)} \psi_{2 n-1}^{-}(E)=\lim _{E \rightarrow \min \left(E_{-}, E_{+}\right)} \psi_{2 n-1}^{+}(E)=+\infty
$$

Further by proposition $(5 . \S I I I-2), \psi_{2 n-1}^{+}(E)$ (resp. $\left.\psi_{2 n-1}^{-}(E)\right)$ is strictly increasing on $\left(0, \min \left(E_{-}, E_{+}\right)\right)$. As required by a.

Let us consider now the equation (66). By setting

$$
\psi_{2 n}(E)=\frac{2 n(p-1)^{\frac{1}{p}}}{p^{\frac{1}{p}} \pi} T_{+}(E)+\frac{2 n(p-1)^{\frac{1}{p}}}{p^{\frac{1}{p}} \pi} T_{-}(E)
$$

And using a similarly argument as above, we have:

$$
\lim _{E \rightarrow 0_{+}} \psi_{2 n}(E)=\bar{\lambda}_{2 n}^{\frac{1}{p}}
$$

And

$$
\lim _{E \rightarrow \min \left(E_{-}, E_{+}\right)} \psi_{2 n}(E)=+\infty
$$

Further, $\psi_{2 n}$ is strictly increasing on $\left(0, \min \left(E_{-}, E_{+}\right)\right)$as required by $b$. 
II The proof will very much resemble the above proof.

By hypotheses $\bar{H}_{7}$, one has $E_{+}=E_{-}=\bar{E}$, and then

$$
T_{-}(E)=T_{+}(E)
$$

Using again proposition $(4 . \S I I)$, we have

$$
\lim _{E \rightarrow \bar{E}} T_{+}(E)=\int_{0}^{a}\{F(a)-F(u)\}^{-\frac{1}{p}} d u<+\infty
$$

And this yields that the range of $\psi_{2 n-1}^{+}(E)$ (resp. $\left.\psi_{2 n-1}^{-}(E)\right)$ is the interval $\left[\bar{\lambda}_{2 n-1}^{\frac{1}{p}}, \mu_{2 n-1}^{\frac{1}{p}}\right]$. Similarly, one shows that the range of $\psi_{2 n}(E)$ is the interval $\left[\bar{\lambda}_{2 n-1}^{\frac{1}{p}}, \mu_{2 n-1}^{\frac{1}{p}}\right]$.

Further, by the $(5 . \S I I I)$ the functions $\psi_{2 n-1}^{+}(E), \psi_{2 n-1}^{-}(E)$ and $\psi_{2 n}$ are strictly increasing on $[0, \bar{E}]$.

Finally, by summing the results collecting above, one sees that there is a collection of infinite sequences of value $\left\{\lambda_{n}^{\frac{1}{p}}\right\},\left\{\mu_{n}^{\frac{1}{p}}\right\} ; n=1,2, \ldots$

Let us now study the link which may exist between those intervals whose ends points are the elements of the sequences of values defined above.

One should ask the following question:

Might one have

$$
\mu_{n}^{\frac{1}{p}} \leq \bar{\lambda}_{n+1}^{\frac{1}{p}} \quad \forall n ?
$$

By using the expression of $\mu_{n}$ and $\bar{\lambda}_{n+1}$, one obtains (after computations)

$$
\frac{n}{p^{\frac{1}{p}}} T(F) \leq(n+1) \frac{1}{p \alpha^{\frac{1}{p}}} B\left(\frac{1}{p}, 1-\frac{1}{p}\right)
$$

Then for fixed $p$, let us check for the value of $n$ for which the above inequality is satisfied.

After some computations, one obtain

$$
n \leq \frac{1}{p^{\frac{p-1}{p}} \alpha^{\frac{1}{p}} \frac{T(F)}{B\left(\frac{1}{p}, 1-\frac{1}{p}\right)}-1}=r(p, F)
$$

This leads us to distinguish two cases: 
First case: $r(p, F)<1$

Under this case, one sees that the inequality (68) is never satisfied. That is we have always.

$$
\bar{\lambda}_{n+1}^{\frac{1}{p}}<\mu_{n}^{\frac{1}{p}}, \quad n=1,2, \ldots
$$

That is the set of interval $I_{n}$ are overlapping; hence we deduce tha final desired results of first case $I$.

Second case: $r(p, F) \geq 1$

In this case, one has always

$$
\mu_{n}^{\frac{1}{p}} \leq \bar{\lambda}_{n+1}^{\frac{1}{p}}, \quad n=1,2, \ldots,[r(p, F)]
$$

and

$$
\bar{\lambda}_{n+1}^{\frac{1}{p}}<\mu_{n}^{\frac{1}{p}} \quad \forall n \geq[r(p, F)]+1
$$

From which, we deduce the desired results of II (2 case).

Let us confine attention for the moment to the singular solutions. Let us recall that they correspond to the case where $E=\bar{E}$, hence they appear every time $\lambda=\mu_{n}$, where $\mu_{n}$ are defined previously by (63). Referring to the below figure which shows a heteroclinic trajectory associated to $\overline{\bar{E}}$ in the phase-plane $(u, w)$ : 


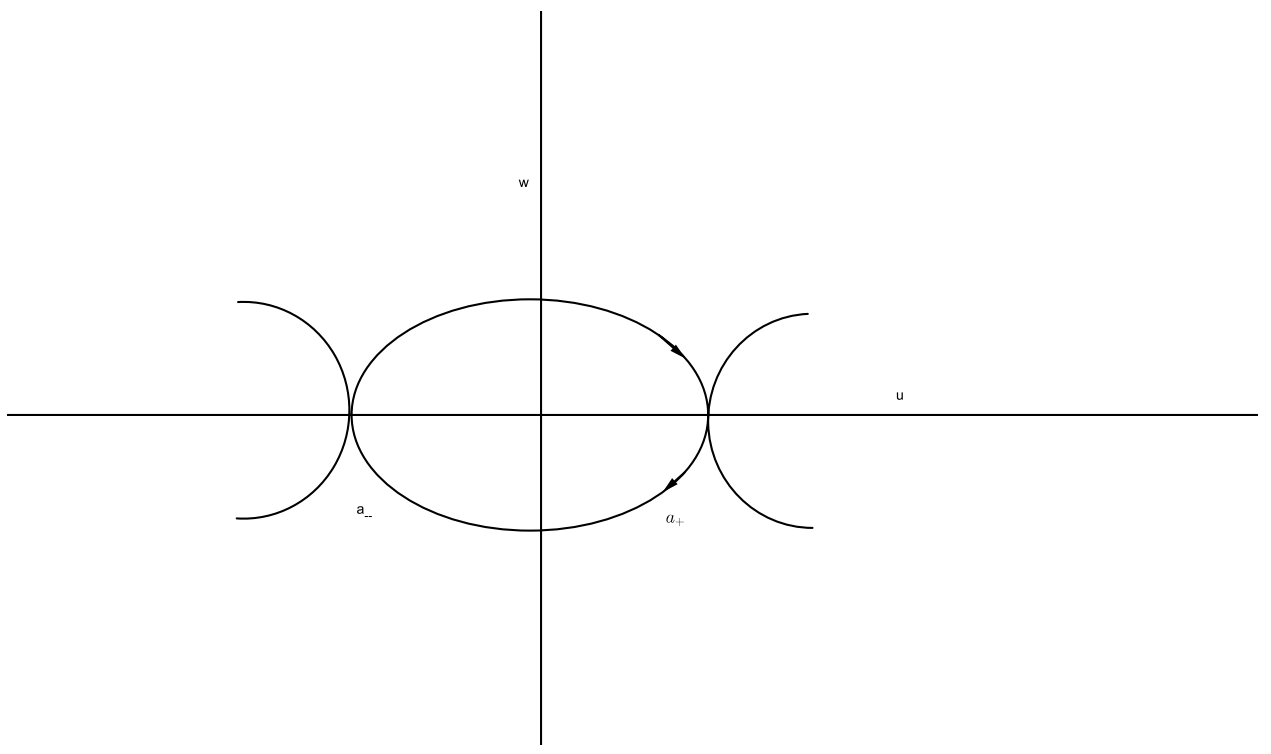

Figure 17: 
Let put

$$
\lim _{E \rightarrow \bar{E}} l^{+}(E)=L^{+}=\lim _{E \rightarrow \bar{E}} l^{-}(E)
$$

Let us firstly consider the solutions which correspond to a half tour.



Figure 18:

Thus, we notice that all a continuum of solutions $\varepsilon_{1}$ take place; it is diffeomorphic to $[0, \pi]$ because one can index it by the parameter $\alpha$, which is the time that the representative point $(u(t), v(t))$ may put in the equilibrium point $(a, 0)$ :

$$
\alpha \in\left[0, \lambda^{\frac{1}{p}} \pi-2 L_{+}\right], \text {with } \lambda>\mu_{1}
$$

So $\varepsilon_{1}$ is diffeomorphic to $[0, \alpha]$ with $0 \leq \alpha \leq \lambda^{\frac{1}{p}} \pi-2 L_{+}$ Let us consider the solutions which correspond to a one tour

Thus, we see this time, that all a continuum of solution $\varepsilon_{2}$ take place, which is diffeomorphic to $[0, \pi]^{2}$, for one can index $\varepsilon_{2}$ by parameters $\alpha$ and $\beta$. So by continuing on, one sees that every time $\lambda=\mu_{n}$, a continuum $\varepsilon_{n}$ of solutions which is diffeomorphic to $[0, \pi]^{n}$ appears.

By collecting all the informations above, we can state the following theorem:

First case A: $1<p \leq 2$

\section{Theorem 1}

i- If $\lambda \leq \frac{\lambda_{1}}{\alpha}$, then problem $\left(E_{\lambda}^{p}\right)$ has only the trivial solution 


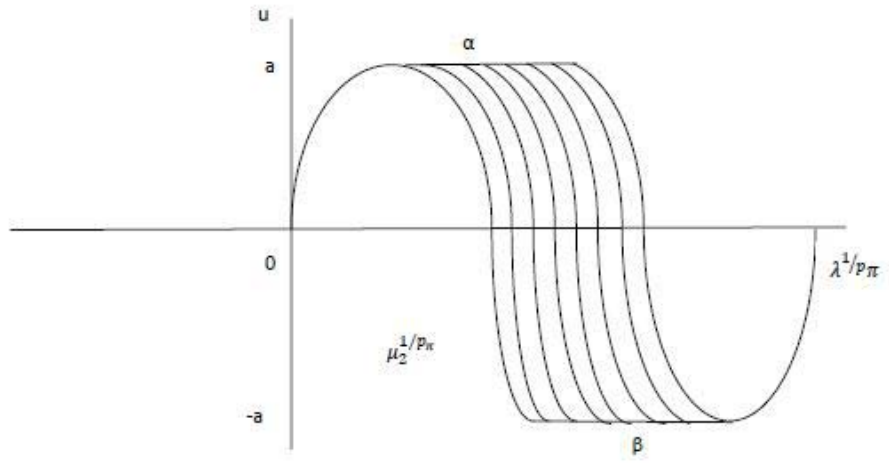

Figure 19:

ii- If $\frac{\lambda_{n}}{\alpha}<\lambda \leq \frac{\lambda_{n+1}}{\alpha}, \quad n=1,2, \ldots$ then problem $\left(E_{\lambda}^{p}\right)$ has precisely $2 n+1$ solutions where $\lambda_{n}$ is the eigenvalue of $\left(\wp(\lambda)\right.$ and $\alpha$ is given by $\left.\bar{H}_{3}\right)$

Thus under the first case A. The bifurcation picture is as portrayed in figure 19.

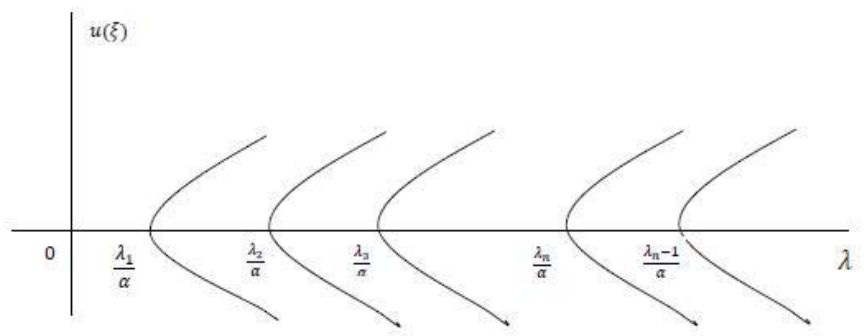

Figure 20: where $u(\xi)=\max _{x \in[0, \pi]}|u(x)|$ 
Second case B: $p>2$

\section{Theorem 2}

i- If $\lambda \leq \frac{\lambda_{1}}{\alpha}$ then problem $E_{\lambda}^{p}$ has only the trivial solutions

ii- If $\frac{\lambda_{1}}{\alpha}<\lambda \leq \frac{\lambda_{n+1}}{\alpha}, \quad n=1,2, \ldots$

then problem $E_{\lambda}^{p}$ has precisely $2 n+1$ regulars solutions and no singular solutions:

$$
u_{0}(x) \equiv 0, \quad u_{n}^{ \pm}(x) \text { where } \frac{d u_{n}^{+}}{d x}(0)>0, \quad \frac{d u_{n}^{-}}{d x}(0)<0
$$

and $u_{n}^{ \pm}(x)$ has exactly $n-1$ simple zeros in $(0, \pi)$.

Thus under the second case $B$ and the statements $i$ ) or $i i$ ). The bifurcation picture is as portrayed in figure 20

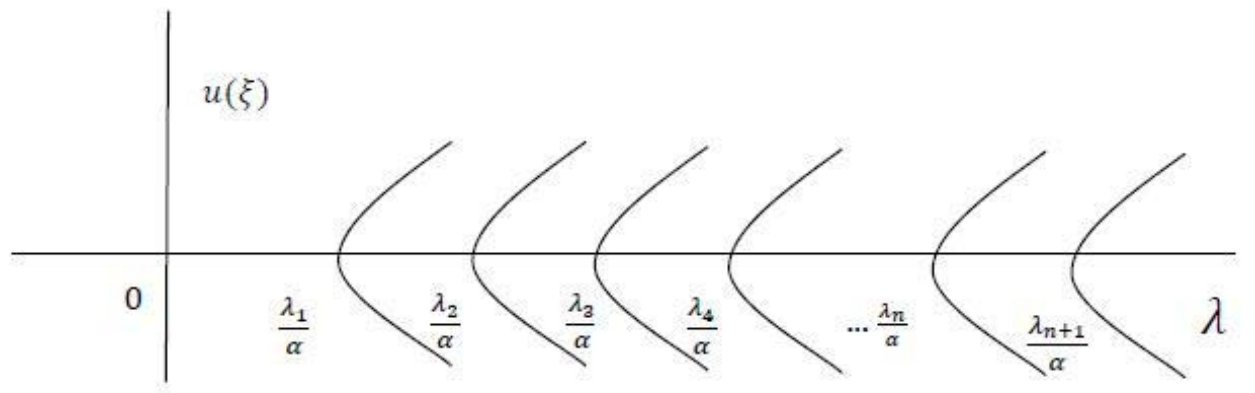

Figure 21: where $u(\xi)=\max _{x \in[0, \pi]}|u(x)|$

We notice that under the first case $A$ and under the second case $B$ the statements $i$ ) or $i i)$, the problem $E_{\lambda}^{p}$ behaves likes in the linear case $(p \leq 2)$.

Under the second case $B$, and $i i i$ ), we shall distinguish two cases depending on whether:

$$
r(p, F)<1 \quad \text { or } \quad r(p, F) \geq 1
$$

First case: $r(p, F)<1$.

We obtain 


\section{Theorem 3}

i- If $\lambda \leq \frac{\lambda_{1}}{\alpha}$

Then problem $E_{\lambda}^{p}$ has only the trivial solution;

ii- If $\frac{\lambda_{1}}{\alpha}<\lambda<\frac{\lambda_{2}}{\alpha}$ Then problem $E_{\lambda}^{p}$ has precisely three regular solutions and no singular solutions;

iii- If $\frac{\lambda_{n+1}}{\alpha}<\lambda<\mu_{n} n=1,2, \ldots$

Then problem $E_{\lambda}^{p}$ has precisely five regulars solutions

iv If $\mu_{n}<\lambda \leq \frac{\lambda_{n+2}}{\alpha} n=1,2, \ldots$.

then problem $E_{\lambda}^{p}$ has precisely three regular solutions and each time $\lambda=$ $\mu_{n}$ there exists a secondary bifurcating continuum $\varepsilon_{n}$ of non trivial singular solutions which is diffeomorphic to $[0, \pi]^{n} n=1,2, \ldots$ where

$$
\mu_{n}=\frac{2^{p} n^{p}(p-1)}{\pi^{p} p}\left(\int_{0}^{a}\{F(a)-F(u)\}^{-\frac{1}{p}} d u\right)^{p}
$$

Thus under statements $i i i$ ) and under the second case $B$ the bifurcation picture is as portrayed in figure 23

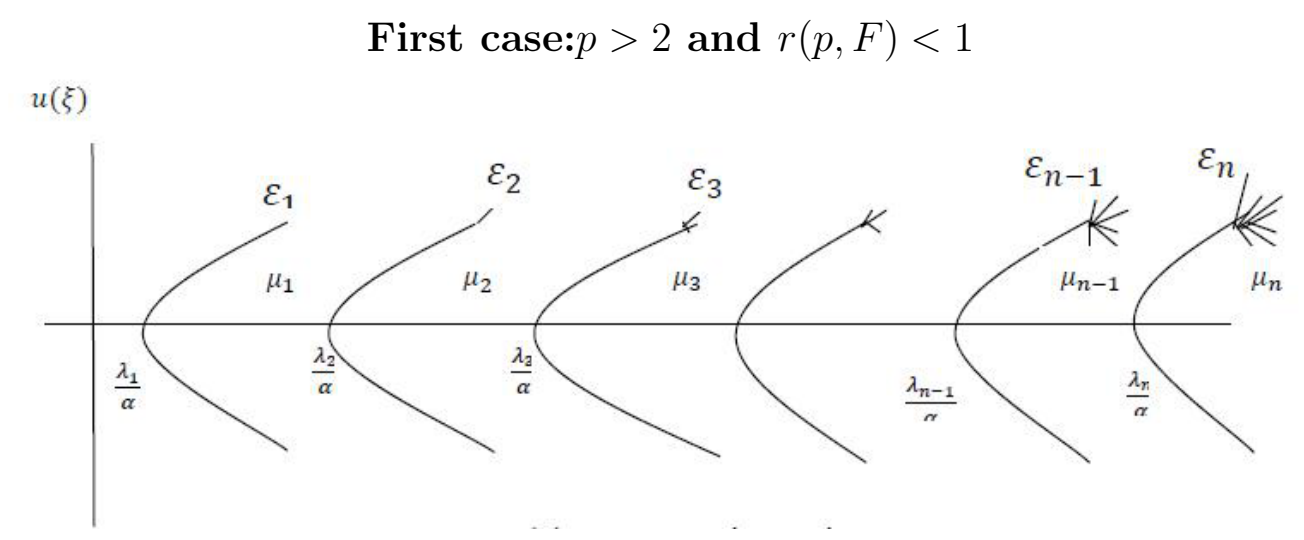

Figure 22: $u(\xi)=\max _{x \in[0, \pi]}|u(x)|$

Second case: $r(p, F) \geq 1$

in this case, we have: 


\section{Theorem 4}

i- If $\lambda \leq \frac{\lambda_{1}}{\alpha}$,

Then problem $E_{\lambda}^{p}$ has only the trivial solution;

ii- If $\frac{\lambda_{n}}{\alpha}<\lambda<\mu_{n} \quad n=1,2, \ldots, r[r(p, F)]$ Then problem $E_{\lambda}^{p}$ has precisely three regular solutions.

iii- If $\mu_{n}<\lambda \leq \frac{\lambda_{n+1}}{\alpha} n=1,2, \ldots,[r(p, F)]$

Then problem $E_{\lambda}^{p}$ has no regular solutions (has only the trivial solution), on the other hand they are apparition of continuum $\varepsilon_{n}$ of singular solutions which is diffeomorphic to $[0, \pi]^{n} n=1,2, \ldots$

iv If $\frac{\lambda_{n+1}}{\alpha}<\lambda<\frac{\lambda_{n+2}}{\alpha} n>n^{*}=[r(p, F)]$

Then problem $E_{\lambda}^{p}$ has precisely three regular solutions

$\mathbf{v}$ If $\frac{\lambda_{n+1}}{\alpha} \leq \lambda<\mu_{n} n>n^{*}=[r(p, F)]$

Then problem $E_{\lambda}^{p}$ has precisely five regular solutions.

vi- $\quad$ And if $\mu_{n}<\lambda \leq \frac{\lambda_{n+2}}{\alpha} n=n^{*}+1, \ldots$.

Then problem $E_{\lambda}^{p}$ has precisely three regulars solutions. And each time $\lambda=\mu_{n}$, there exists secondary bifurcating continuum $\varepsilon_{n}$ of singularities of non trivial solutions which is diffeomorphic to $[0, \pi] n=1,2, \ldots$.

Thus under statements of theorem 4 the bifurcation picture is as portrayed in the following figure

Second case: $p>2$ and $r(p, F) \geq 1$

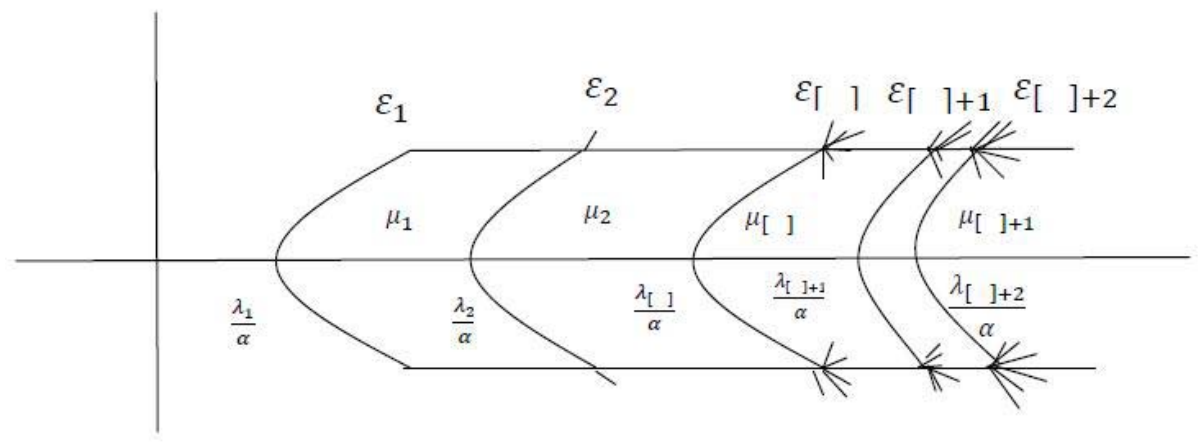

Figure 23: where []$=[r(p, F)], u(\xi)=\max _{x \in[0, \pi]}|u(x)|$ 


\section{Example of application}

a) In what follows we shall give an example of function $f$ which permits us to determine explicitly the integer $[r(p, F)]$ defined previously.

Let us consider the following function

$$
f(u)=-|u|^{2(p-1)} u+|u|^{p-2} u, \quad p>2
$$

One can easily check that this function satisfies all the hypotheses $\left.\bar{H}_{1}\right), \ldots, \bar{H}_{6}$, $\bar{H}_{7}$ whith

$$
\lim _{u \rightarrow 0} \frac{f(u)}{|u|^{p-2}} u=\alpha=1, \quad a=1
$$

In other hand one has

$$
\begin{aligned}
T(F) & =\int_{0}^{1}\{F(1)-F(u)\}^{-\frac{1}{p}} d u \\
& =\int_{0}^{1}\left\{\frac{1}{2 p}+\frac{1}{2 p} u^{2 p}-\frac{1}{p} u^{p}\right\}^{-\frac{1}{p}}
\end{aligned}
$$

In order to investigate the behavior of this number with respect to $p$, we make the following change of variables:

$$
u^{p}=X
$$

We get after some easy computation:

$$
T(F)=\frac{1}{p} \int_{0}^{1}\left\{\frac{1}{2 p}+\frac{1}{2 p} X^{2}-\frac{1}{p} X\right\}^{-\frac{1}{p}} X^{\frac{1-p}{p}} d X
$$

Furthermore, if we put

$$
\frac{1}{2 p} X^{2}-\frac{1}{p} X+\frac{1}{2 p}=Y
$$

We deduce from this equation that

$$
X=1-p \sqrt{\frac{2}{p} y}
$$

And after some computation one obtains:

$$
\begin{aligned}
T(F)= & \left(\frac{2 p}{p}\right)^{\frac{1}{p}} \int_{0}^{1}(1-y)^{\frac{1-p}{p}} y^{-\frac{2}{p}} d y \\
& =\left(\frac{2 p}{p}\right)^{\frac{1}{p}} B\left(\frac{1}{p}, 1-\frac{2}{p}\right)
\end{aligned}
$$


Which one can write it again by using of the Beta function in the following manner

$$
T(F)=\frac{(2 p)^{\frac{1}{p}}}{p} \frac{\Gamma\left(1-\frac{2}{p}\right)}{\left(\Gamma\left(1-\frac{1}{p}\right)\right)^{2}} B\left(\frac{1}{p}, 1-\frac{1}{p}\right) ; p>2
$$

Hence the integer $r(p, F)$ defined previously becomes in this particular:

$$
r(p, F)=\frac{\left(\Gamma\left(1-\frac{2}{p}\right)\right)^{2}}{2^{\frac{1}{p}} \Gamma\left(1-\frac{2}{p}\right)-\left(\Gamma\left(1-\frac{1}{p}\right)\right)^{2}}
$$

By considering this real number as a function of $p$, the curve $v=r(p, F)$ is plotted in the following figure:



Figure 24: where []$=[r(p, F)], u(\xi)=\max _{x \in[0, \pi]}|u(x)|$ 
We see effectively that there exists a unique real number $p^{*}>2$, such that:

$$
\begin{gathered}
r(p, F)<1 \quad \forall p \in\left(2, p^{*}\right) \\
r(p, F) \geq 1 \quad \forall p \geq p^{*}
\end{gathered}
$$

On the other hand, one can easily prove by using the properties of the beta function that: the integer $[r(p, F)]$ tends to infinity when $p$ goes to infinity.

b) Characterization of regular solutions associated to heteroclinic trajectory.

As the heteroclinic trajectory is closed, one deduce that the solution $u_{n}$ is periodic, the period $T_{n}$ is given by

$$
T_{n}=4\left(\frac{p-1}{p \mu_{n}}\right)^{\frac{1}{p}} l(F)
$$

From the fact that $\mu_{n}=\frac{2^{p}\left(n^{p}(p-1)\right)}{\mu^{p} p} l^{p}(F)$, on deduce that

$$
T_{n}=\frac{2 \pi}{n}, \quad n=1,2, \ldots
$$

In this case we note that the period of the motion is independent of the amplitude. Furthermore the zeros of the functions $\mu_{n}$ are given by the formula:

$$
x_{n}^{j}=j \frac{\pi}{n} \quad j=0,1,2, \ldots, n
$$

\section{References}

[1] M.Amattat.Doctorat thesis. Bruxelles 1988.

[2] A note on a magnetohydrodynamics cowette flow. Int.J. of Applied Mechanics and Engineering, 2010, vol15, N4,pp.1287 - 1295.

[3] P.Binding and P.Drabek, sturm-Liouville theory for the p-Laplacian, Studia Sci. Math.Hungar. 40(2003), 375 - 396.

[4] P.A. Bining, B.P.Rynne, Half-eigenvalues of periodic Sturm-Liouville problem, J. Differential Equation 206(2004), 280 - 305.

[5] P.A. Bining, B.P.Rynne, The spectrum of the periodic p-Laplacian, J. Differential Equns. 235(2007), $199-218$.

[6] N.CHAFFEE.E.F.INFANTE. A bifuracation problem for a nonlinear partial differential equation. Applicable analysis 4(1974)17 - 37. 
[7] Diaz, J.I: Nonlinear Partial Differential Equations and Free Boundaries. Volume 1 Elliptic Equations, Pitman, Boston(1985).

[8] Pavel Drabek and Stephen B.Robinson, Resonance problems for the pLaplacian, J. Funet. Anal. 169(1999), no. 1, 189 - 200. MR 1726752(200j : 35096), http://dx.doi.org/10.1006:jfan.1999.3501.

[9] P.DRABEK. Asymptotic bifurcation problems for quasilinear equation. Existence and multiplicity results. Topological methods in nonlinear analysis journal of the juliusz schauder volume 25, 2005, $183-184$

[10] P.DRABEK THE p-LAPLACIAN6MASCOT OF NONLINEAR ANALYSIS Acta Math.Univ. Comenianae Vol. LXXVI, 1(2007)? PP. 85 - 98 Proceedings of Equadiff 11.

[11] Pavel Drabek and Stephen B.Robinson, Resonance problems for the pLaplacian, J. Funet. Anal. 169(1999), no. 1, 189 - 200. MR 1726752(200j : 35096), http://dx.doi.org/10.1006:jfan.1999.3501.

[12] Hartman P.(1964): Ordinary Differential Equation-News York: Wiley.

[13] B.P.Rynne, p-laplacian problems with jumping nonlinearities, J.differential equation 226(2006), $501-524$.

[14] B.P.Rynne, spectral properties and nodal solutions for second order, mpoint, boundary value problems, Nonlinear Analysis 67(2007), 3318-3327.

[15] B.P.Rynne, NONRESONANCE CONDITIONS FOR GENERALISED $\varphi$-LAPLACIAN PROBLEMS WITH JUMPING NONLINEARITIES J.Differential Equns.

[16] L.Veron, M. Guedda, bifurcation phenomena associted to the p-laplace operator. Tran.am.math. SOC, 310, 419643151988).

Received: June 1, 2013 